\title{
The Hypergeometrical Universe: Cosmogenesis, Cosmology and Standard Model
}

\section{Pereira MA*}

Department of Mathematics, The Rutgers University, New Brunswick, NJ, USA

\begin{abstract}
This paper presents a simple and purely geometrical Grand Unification Theory. Quantum Gravity, Electrostatic and Magnetic interactions are shown in a unified framework. Newton's Gravitational Law, Gauss' Electrostatics Law and Biot-Savart's Electromagnetism Law are derived from first principles. Gravitational Lensing, Mercury Perihelion Precession are replicated within the theory. Unification symmetry is defined for all the existing forces. This alternative model does not require Strong and Electroweak forces. A 4D Shock-Wave Hyperspherical topology is proposed for the Universe which together with a Quantum Lagrangian Principle and a Dilator based model for matter result in a quantized stepwise expansion for the whole Universe along a radial direction within a 4D spatial manifold. The Hypergeometrical Standard Model for matter, Universe Topology, Simple Cosmogenesis and a new Law of Gravitation are presented. Type 1A Supernova Survey HU results is provided. A New de-Broglie Force is proposed.
\end{abstract}

Keywords: Electromagnetism law; Hyperspherical topology; Hypergeometrical standard model; Topology; Cosmogenesis; Electrostatics law; Magnetic interactions; Quantum gravity

\section{Introduction}

Grand Unification Theories are the subject of intense research. Among current theories, Superstring, M-Theory, Kaluza-Klein based 5D Gauge Theories have shown diverse degrees of success. All theories try to keep the current conceptual framework of science. Kaluza-Klein melded both Electromagnetism and Einstein Gravitational equations in a $5 \mathrm{D}$ metric [1].

Instead of concentrating in keeping the current formalism, this work concentrates on what to say, the conceptual framework of Nature instead. All the common constructs: mass, charge, color, hypercharge are dropped in favor of just dilator positions and dilaton fields, which are local metric modulators and traveling modulations, respectively. There is no need for the concepts of charge or mass. Inertial Mass is modeled as a quantity proportional to the $4 \mathrm{D}$ metric displacement volume at precise phases of de-Broglie cycles. These are the footprints of the dilator on our 3D Universe. Charge sign is modeled by dilator phase (sign) on those specific phases. The mapping is needed to demonstrate that the geometrical framework replicates current scientific knowledge.

As we search for more encompassing theories, they became increasingly complex and speculative. Current best candidate to explain cosmology is the Lambda-CDM (Lambda Cold Dark Matter) based on Friedmann-Lemaitre variation of General Relativity [2]. It is an extension of Einstein's equations to accommodate an expanding (dizzyingly inflating, accelerating, slowing down, accelerating again) Universe as perceived by Redshifted-Stellar-Candles-mapped distances. To explain such complex dynamics, Lambda-CDM equation below contains 6 parameters, excluding $H_{0}$. $\mathrm{HU}$ does that without a single parameter.

$$
H(a) \equiv \frac{\dot{a}}{a}=H_{0} \sqrt{\left(\Omega_{c}+\Omega_{b}\right) a^{-3}+\Omega_{r a d} a^{-4}+\Omega_{k} a^{-2}+\Omega_{D E} a^{-3(1+w)}}
$$

Current interpretation of type 1A Supernova distances produces an unyielding Universe where linear distances of more than 40 billion light years were observed on a 13.58 billion years old universe. Inflation Theory was created to explain the unexpected observations. $\mathrm{HU}$ provides a much simpler view of events [3]. HU proposes that
Supernova distances might be overestimated by $G^{\frac{3}{2}}$, where $\mathrm{G}$ is the Gravitational Constant, due to Chandrasekhar mass $\mathrm{G}$ dependence.

Supernova Analysis Section presents HU Cosmology as well as the reproduction of the current view of Inflation and Expansion processes to demonstrate how the possible misreading of distances can lead to the current unexpected conclusions.

In the HU topology section the three spatial coordinates are mapped to a lightspeed expanding hyperspherical hypersurface, thus introducing a new spatial dimension the radial dimension. To be consistent with light speed for short-distance measurements, the $4 \mathrm{D}$ speed of light should be adjusted to be. We will see in the Supernova Analysis Section that the effective speed of light changes with distance when measuring ancient photons. In $\mathrm{HU}$, ancient photons slow down as they come closer to us. A clear geometric argument is presented.

A geometric reason for redshift, the Cosmic Microwave Background [4] and its homogeneity is provided.

HU introduces an absolute frame of reference "the fabric of space (FS), which cannot be measured from within the 3D hypersurface. Local deformation of FS is associated with velocity. Only relative deformational states (relative velocities) are measurable. Local rate of deformation of FS is associated with acceleration and thus with Force. $\mathrm{HU}$ also provides the reason why $\mathrm{c}$ is the limiting velocity and why there is inertial motion.

A simple Cosmogenesis model is presented on the Cosmogenesis section.

*Corresponding author: Marco Pereira, Department of Mathematics, The Rutgers University, New Brunswick, NJ, USA, Tel: +1 848-932-7496; E-mail: marco.pereira@rutgers.edu

Received May 09, 2016; Accepted December 15, 2016; Published December 28, 2016

Citation: Pereira MA (2016) The Hypergeometrical Universe: Cosmogenesis, Cosmology and Standard Model. J Generalized Lie Theory Appl 10: 248. doi:10.4172/1736-4337.1000248

Copyright: (c) 2016 Pereira MA. This is an open-access article distributed under the terms of the Creative Commons Attribution License, which permits unrestricted use, distribution, and reproduction in any medium, provided the original author and source are credited. 
On the Cosmological Coherence section, the consequences of the topology of the Hypergeometrical Universe and the homogeneity proposed in the Fundamental Dilator based model for matter is shown to result in a cosmological coherence, that is, the whole 3D universe expands radially at light speed and in de-Broglie (Compton) steps.

When cosmological coherence is mentioned it is within the framework of absolute time and absolute $4 \mathrm{D}$ space (RXYZ or $\Phi \mathrm{XYZ}$ ). There is no sense in speaking of synchronous motion within frameworks containing proper time $\tau$. For simplicity, all force derivations are done considering a framework at rest with respect to the Fabric of Space.

A new Quantum Lagrangian Principle (QLP) is created to describe the interaction of dilators and dilatons, Quantum gravity, electrostatics and magnetism laws are derived subsequently as the result of simple constructive interference of five-dimensional spacetime wave overlaid on an expanding hyperspherical universe. In the electrostatics and magnetism derivation, a 4D-mass of a Hydrogen Mass a.m.u. electron or fat electron is used. This means that dilatons (5D spacetime waves driven by coherent metric modulations) are coherently produced by all phases of the dilator coherence.

The 4D-Mass will be fixed to match the 3D-Mass. This will provide us information about the anisotropy of space in the form of an effective Planck Constant for gravitational and electromagnetic dilaton fields. This sheds light on how flush and perpendicular states effect dilaton waves. Space is anisotropic due the existence of our Universe in the traveling hypershell. The tangential spring constant is the Planck Constant and tangential waves are the de-Broglie waves. Hypervolumetric waves carry Electromagnetism and Gravitation as well as Light.

Currently, we consider Black Holes to be a gravitationally induced space deformation where not even light can escape. This creates a logical flaw since Black Holes are made up by matter aggregates. Unless Matter is described in terms of space, things are not properly tied down or they might be tied down in a Rube Goldberg manner.

Among Particle Physics strongest challenges was the inability of observing single quarks out of collisional experiments. No matter how strong the collision, single quarks were nowhere to be seen. StrongForce was introduced to explain the unexpected observation. It would grow to infinity rapidly as the quark separation increases. Quark closeness would bring asymptotic freedom.

New quantum numbers (internal dimensions) were added to accommodate unexpected new particles. Particle Physics observables are both physical (energy, scattering angle, dipole, quadrupole moments, spin) and relational (part of a decay chain reaction or family) [4].

Quarks' existence was inferred from structured scattering, that is, scattering from which one can infer internal structure from the angular distribution of products plus the quantum number based particle taxonomy. What if the observed structure were akin to an excited state of Matter (or space if Matter is described in terms of space deformations)? One can relate to that if one considers atomic collisions. Depending on the energy of the collision, scattering will contain information about excited state orbitals. In analogy to Quarks, one cannot separate the geometric building blocks of those potential excited orbitals. Somehow when considering molecular dynamics collision, nobody proposed the existence of Molecular Quarks to explain products channels. This might seen like a facetious comment, but it is not. This is to emphasize that Quarks is not the only solution to structured scattering.

Due to the success of the Standard Model in explaining scattering events, one doesn't expect HU to start from scratch. One would expect HU to provide a simple map that would link Quarks, Hyperons to structured fluctuations (deformational coherences) of space. HU would provide guidance to modify parts of the Standard Model that are in conflict.

Since HU is derived within a Spatial Stress-Strain paradigm, there is no need for a mechanism from which to derive mass. Conversely, HU provides the mass for the four fundamental particles associated with the Fundamental Dilator (proton, electron, antiproton, positron).

Hypergeometrical Universe Theory model for matter is based on the Fundamental Dilator (FD). This is a coherence between stationary deformation states of the local metric, where local was used to emphasize localization.

These coherences do not extend over long distances and have in fact, very small cross-sections. FD excited states are associated with the hyperon family and they have structure or topology.

This might explain internally structured scattering results and the inseparability of those internal components. This means that $\mathrm{HU}$ associates internal composition to coherences between excited deformational states of the local metric.

The decaying of these excited states gives rise to the decay chain reactions. From assignment to simple dilator excited states (neutron, pion) and decay reactions one can stepwise build the whole taxonomy. Since particles are assigned to coherences of deformation of space, this fits well with a Black Hole being made of deformed space.

Coherences produce shape-shifting space deformation and thus space deformation waves (dilaton field). The agent of deformation is called a dilator. The resulting waves are the dilaton field.

This is an ongoing line of research. Very early results will be provided to show the potential of the model.

A grand unification theory is a far-reaching theory and touches many areas of knowledge. Arguments supporting this kind of theory must be equally scattered. Many arguments will be presented with little discussion when they are immediate conclusions of the topology or simple logic.

\section{Hypergeometrical Universe Topology}

The universe direction along $\mathrm{X}$ is represented by the band. $\mathrm{X}$ (or $\mathrm{Y}$ or $\mathrm{Z}$ ) is displayed along the perimeter of the circle. The circle radius is equal to the age of the universe times the speed of light. Also, shown in the diagram are $\Phi$ (cosmological time), proper time $\tau$, radial direction $\mathrm{R}$, proper radial projection $\mathrm{r}$, the Cosmological Angle $\alpha$ between two reference frames $\mathrm{XYZ} \tau$ and $\mathrm{X}^{\prime} \mathrm{Y}^{\prime} \mathrm{Z}^{\prime} \tau^{\prime}$, the local torsion angles and $\alpha \mathrm{R}$. By choosing a local metric for xyz $\tau$ Minkowskian and having a Lorentz transformation to relate $\mathrm{XYZ} \tau$ and $\mathrm{X}^{\prime} \mathrm{Y}^{\prime} \mathrm{Z}^{\prime} \tau$ ' reference frames, one can assure that the theory obeys Strict Relativity.

The universe is hypothesized to be created by a four-dimensional explosion, a Big Bang in a Four-Dimensional Spatial Manifold. The evolution of such Big Bang is a lightspeed expanding three-dimensional hypersurface on quantized de-Broglie steps. The steps have length close related to the Compton wavelength associated with the gravitational fundamental dilator (the atomic mass of a hydrogen atom). It will be shown in Table 1:

\section{Definitions}

1. Cosmological time $\Phi$ represents an absolute time frame, as 


\begin{tabular}{|c|c|}
\hline Dilator & 4D-Mass(a.m.u.) \\
\hline Gravitational Fundamental Dilator (GFD) & Hydrogen Mass x 2 \\
\hline Electronic Fundamental Dilator (EFD) & Hydrogen Mass \\
\hline
\end{tabular}

Table 1: Fundamental dilators.

envisioned by Newton and Mach - it is a fifth dimension in the Hypergeometrical Universe Model. It times the expansion of the Universe.

2. Proper time $\tau, \tau^{\prime}$ are projections of the Cosmological Time $\Phi$ (which is always along a radial direction) on the respective reference frames.

3. Fabric of Space (FS) is the Lightspeed traveling locus where our 3D Universe exists. This is a 3D hypersurface of a shockwave within a 4D spatial manifold. Anything at rest with respect to the Fabric of Space would just travel radially at the speed of light. At the Big Bang, all dilators would be initially traveling at the speed of light, not only radially but also tangentially in all directions. When the Universe is a point, there is no difference between tangential and radial directions. As the Universe aged, dilators would, on average, reach equilibrium and a low (zero) velocity with respect to FS.

4. The radial direction is a preferential direction in $4 \mathrm{D}$ space. It is the radial expansion direction. This direction doubles as a direction on $4 \mathrm{D}$ Space and a projection of the cosmological time, since they are related by the expansion speed (lightspeed).

5. $\alpha_{\tau}$ and $\alpha_{r}$ represent both a direction of propagation and a deformation of the local fabric of space. Since these angles point to direction of propagation, a local deformation of the fabric of space maps directly to a state of motion. Motion is the result of the relaxation process of the local FS (Hypergeometrical Universe interpretation of Newton's first law) as the FS expands.

6. XYZR is modeled as a Cartesian space.

7. $x y z \tau$ (proper reference frame) is modeled as a hyperbolic space and thus consistent with Strict Relativity [5-7] if one considers that the Lorentz transformation is a rotation on an imaginary angle equal to $\operatorname{atan}(\mathrm{v} / \mathrm{c})$.

\section{Universe expansion and the Hubble constant}

Edwin Hubble [8] discovered that Stars and Galaxies are receding from us at speeds that increase linearly with distance. Below is Hubble Law governing the velocity $\mathrm{V}$ for objects at distance $\mathrm{L}$ :

$$
V=H_{0} L
$$

From the proposed topology shown in Figure 1 one can easily ascertain the Hypergeometrical Universe model for the Hubble Constant:

$$
H_{0}=\frac{c}{R_{0}}
$$

where $\mathrm{c}$ is the speed of light and $R_{0}$ is the $4 \mathrm{D}$ Radius of the Universe (age of the Universe time the speed of light). For $H_{0}=72 \mathrm{~km} / \mathrm{s} / \mathrm{Mpc}$ :

$$
R_{0}=\frac{c}{H_{0}}=13.58 \text { billionlight }- \text { years }
$$

Yielding the age of the Universe as 13.58 billion years.

Peering into the past takes place through the $4 \mathrm{D}$ spatial manifold and where light is emitted always through a $45^{\circ}$ perspective. The $45^{\circ}$ is due to the light speed expansion of the Universe. The 45 degrees is the angle of emission with respect to the radial line on the hypersphere of

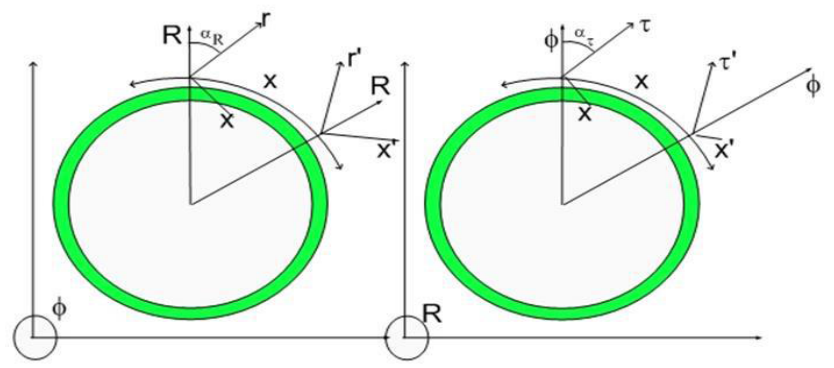

Figure 1: Cross sections of the proposed hyperspherical light speed expanding universe.

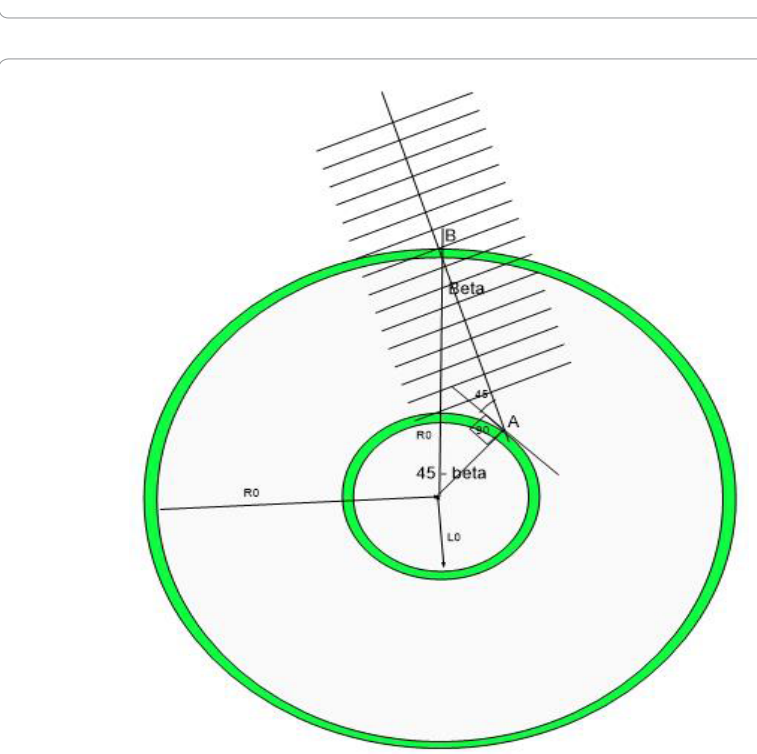

Figure 2: This picture shows how one would see the past in the Hypergeometrical Universe Model.

origin. Light then travels through a Line-Of-Sight path to us as shown in Figure 2.

Light is modeled as a modulation of the dilaton field (seen as a carrier wave) by the varying position of the generating dilator during light emission (oscillating dipole). As the Universe expands, that light interacts with different regions of the 3D Hypersphere. The angle between the k-vector and the local surface perpendicular (radial line) changes as light travels. That is the geometric reason for the Doppler Shift.

Light emission angle with the radial line is always 45 degrees. $\mathrm{K}$-vector direction for the light that we detect is always a line-of-sight vector.

\section{Time-Of-Arrival (TOA) constraint and the 4D speed of light}

If you were to consider any other point in the early epoch shown in Figure 2, the k-vector would had missed us. Light must travel to where we are and arrive when we are there. This is the Time-Of-Arrival (TOA) Constraint.

Maxwell equations indicate that light propagates by inducing a polarization. In the next step, polarization induces electromagnetic field. The resulting cycle is the light propagating on a polarizable medium. HU considers the 3D Hyperspherical Universe to be that polarizable medium. The radial velocity of light is always $c$, since the 
polarizable medium travels at that speed. This means that light speed will adjust itself to different line-of-sight angles automatically and thus satisfy the TOA constraint, if the initial k-vector points directly to us.

Geometric redshifting can also be easily understood from Figure 2 using simple geometry. Notice that the k-vector remains constant while the Fabric of Space changes, tilting as the photon comes closer to us. This tilting means that the velocity projection onto our hyperplane changes. Ancient photons slow down when approaching us. Missing photon momentum is taken by the FS.

\section{Supernova distance analysis}

HU was applied to the Supernova Survey [9]. For short distance one can calibrate the Hubble constant using parallax. Longer distances are calculated using Stellar Candles (type 1A Supernovae explosions).

HU calculates the Gravitational Constant from first principles to be inversely proportional to the $4 \mathrm{D}$ radius (see equation 93 ) of the Universe. This means that prior epoch (smaller hyperspheres) would have stronger gravitation. Since Chandrasekhar Masses of WhiteDwarfs (precursors of Supernovae explosions) are proportional to $G^{-\frac{3}{2}}$, earlier epochs White-Dwarfs would have smaller masses. Since absolute peak luminosity is proportional to the rate of ${ }^{56} \mathrm{Ni}$ formation and that is proportional to the carbon concentration squared, that implies in absolute peak luminosity being proportional to $G^{-3}$. Carbon concentration scales down with Chandrasekhar masses. Notice that this approximation of the nuclear chain reaction by its first step is only modeling the leading edge of the chain reaction. After peak luminosity, ejecta takes over and luminosity becomes the results of a diffusive process. Supernova distances are measured based solely on its absolute peak luminosity (after WLR adjustment).

HU implies that distances are overestimated. Correction of distances and the application of the simple geometric arguments to explain redshift, leads to HU precise predictions without any parametrization. This would explain the perceived Universe acceleration without the need for Dark Energy, since the overestimation gets smaller the closer that epoch is from ours.

After tangentially thermalizing (e.g. 4-Billion-Years-epoch-galaxy) and galaxy formation, masses (center of mass) lose their relative velocity to the FS. Under those conditions, relative motion occurs as they ever did, but the relative distances in terms of cosmological angle stop changing. That 4-Billion-Years-epoch-galaxy distance on today's Universe would lie approximately at 9.58 billion light-years away since its light came from 9.58 billion years ago. Below are HU results to distance vs redshift (Figure 3): where the error is given by (Figure 4):
The topological view of the Supernova explosions is presented in Figure 5: Smooth line are HU line-of-sight predictions. Red-dots represent an ALWAYS 45 degrees w.r.t. radial line photon path. This path would follow the T.O.A. constraint, but would not present any redshift. This path has been discarded because of that.

There are important and subtle issues associated with the HU Supernova Analysis:

1. The derivation of $\mathrm{d}(\mathrm{z})$ was done using line-of-sight optical path. If the velocity of light were constant, then Time-Of-Arrival constraint would not be respected.

Solution: When considering light propagation in a polarizable media, it is easy to remember that Electromagnetic Field creates Polarization $\rightarrow$ which then creates Electromagnetic Field in a continuous cycle.

Since polarizable media (the 3D Universe) is traveling radially at speed c, light will always travel radially at the same speed c, no matter where the k-vector is pointing to. This means that the tangential velocity of light of redshifted (ancient) photons SLOWS DOWN as they approach us.

2. The normalized value for distance $\mathrm{d}(\mathrm{z})$ used is $\frac{R_{0}-R(t)}{R_{0}}$ instead
the real distance.

Solution: HU prescribes that the dilaton field decays with the inverse number of cycles (equation 40-42). The number of traversed cycles (or hyperspheres) between us and any point within an inner hypersphere is always the same. This means that one should use the distance between hyperspheres $\frac{R_{0}-R(t)}{R_{0}}$ as the observed distance! The $\mathrm{k}$-vector orientation depends only upon the relative few de-Broglie steps taking place during the photon emission.

Notice that the photon is emitted at 45 degrees. Subsequently, as the FS tilts, very small adjustments are made to conserve photon momentum at each step. Each de-Broglie step is around 0.19 femtometers and the distance traverse by Supernova explosion photons is millions to billions of light-years, so the amount of adjustment per step is very, very small.

\section{D perceived acceleration}

HU predictions can be used to explain the current view of the Cosmos, including the perceived acceleration, initial Inflation. The HU distance is given by:

$$
D(t)=\frac{R_{0}-R(t)}{R_{0}}=1-a(t)
$$
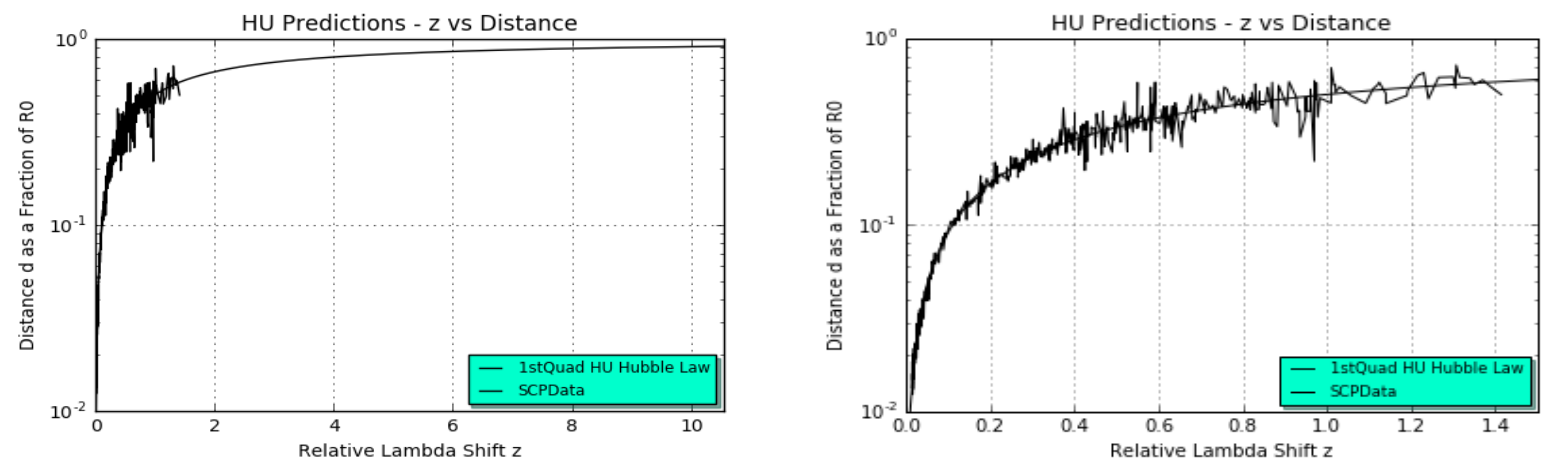

Figure 3: Distance versus redshift. 
Citation: Pereira MA (2016) The Hypergeometrical Universe: Cosmogenesis, Cosmology and Standard Model. J Generalized Lie Theory Appl 10: 248. doi:10.4172/1736-4337.1000248

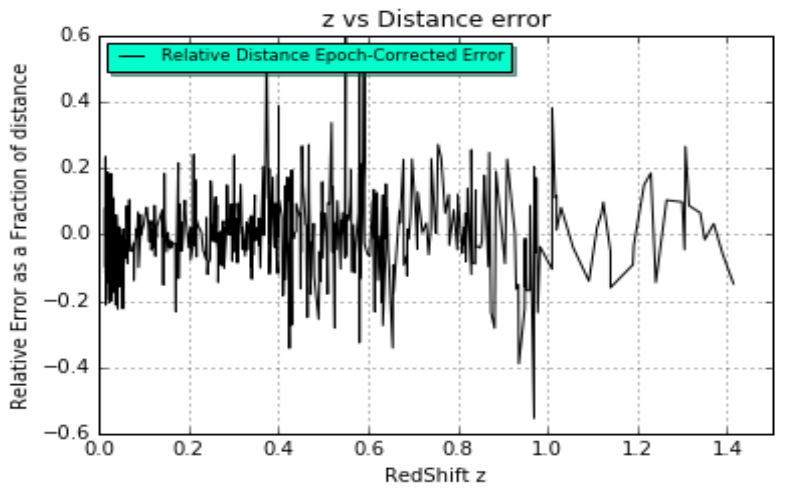

Figure 4: Relative Distance Error for epoch corrected survey distances.
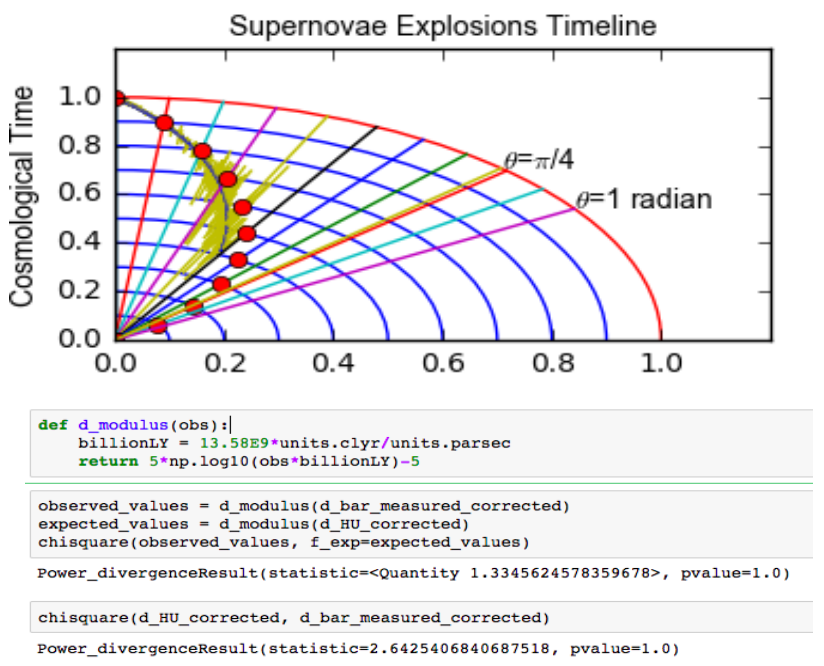

Figure 5: Yellow lines are Supernova explosion (Type 1A Supernova Survey Union 2.1).

where

$$
a(t)=\frac{R(t)}{R_{0}}=\frac{c t}{R_{0}}
$$

That L-CDM distance is overestimated by:

$$
D_{o b s}(t)=1-a_{o b s}(t)=D(t)\left(\frac{R_{0}}{R(t)}\right)^{3 / 2}=(1-a(t)) a(t)^{-3 / 2}=a(t)^{-3 / 2}-a(t)^{-1 / 2}
$$

Taking derivatives:

$$
\begin{aligned}
& \frac{d D_{o b s}(t)}{d t}=\left(-\frac{3}{2} a(t)^{-5 / 2}+\frac{1}{2} a(t)^{-3 / 2}\right) \dot{a}(t) \\
& \frac{d^{2} D_{o b s}(t)}{d t^{2}}=\left(\frac{15}{4} a(t)^{-7 / 2}-\frac{3}{4} a(t)^{-5 / 2}\right) \dot{a}(t)^{2}
\end{aligned}
$$

Since

$$
\frac{d a(t)}{d t}=\frac{c}{R_{0}}
$$

and

$$
\frac{d^{2} a(t)}{d t^{2}}=0
$$

The resulting observed acceleration is given by:

$$
\frac{d^{2} a_{o b s}(t)}{d t^{2}}=\frac{3 c^{2}}{4 R_{0}} a(t)^{-7 / 2}(5-a(t))
$$

Below is the view of the perceived acceleration resulting from possible misreading of Supernovae distances (Figure 6):

Figure 6: Perceived Universe Acceleration in units of $\frac{R_{0}}{(\text { BillionYears })^{2}}$. Left Panel: Inflation Period. Right Panel: Current Universe Acceleration $=\frac{1.6 \%}{(\text { Billion Years })^{2}}$.

If we use units of $\frac{R_{0}}{(\text { BillionYears })^{2}}$ instead:

$$
\frac{d^{2} a_{o b s}(t)}{d t^{2}}=\frac{3}{4(13.58)^{2}} a(t)^{-7 / 2}(5-a(t))=0.4 \% * a(t)^{-7 / 2}(5-a(t))
$$

This means that for current times $(\mathrm{a}(\mathrm{T})=1)$, the Universe has been perceived as accelerating at $\frac{1.6 \%}{(\text { BillionYears })^{2}}$.

Notice that the acceleration becomes negative at $\mathrm{R}(\mathrm{t})=5 R_{0}$. All these results are likely to be artifacts of the overestimation of Supernovae distances.

\section{Simple Cosmogenesis}

Figure 7 is the pictorial display of equilibrium at the incipient Universe (prior to irreversible dimensional phase transition) and the Big Bang irreversible transition.

Top-left displays the Zero-Dimensional Universe (just numbers, adding always to zero), following by the Unidimensional Space (just equal size opposing vectors along a line) and eventually to our 5D Spacetime Universe.

Entropy of a 5D Universe allowed for partial recombination of the initial macroscopic fluctuation, represented by the two states
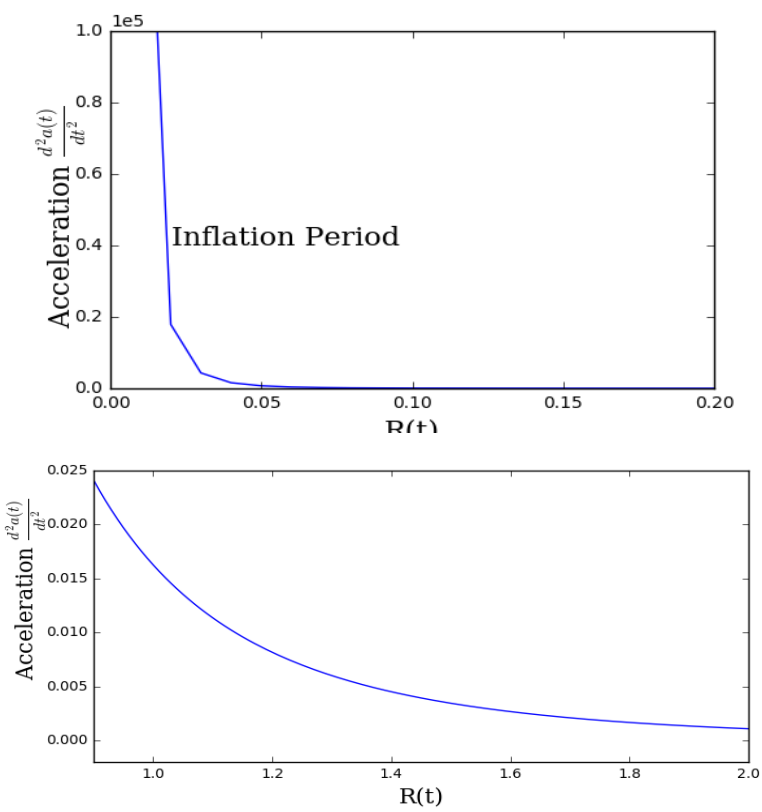

Figure 6: Perceived Universe Acceleration in units of $\mathrm{R}_{0} /$ Billion Years 


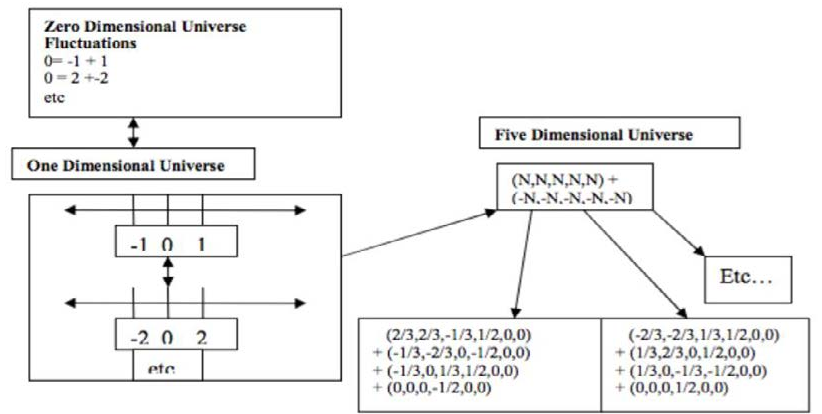

Figure 7: This picture displays the mapping of the different phases of the Universe coming into existence to Mathematical Constructs.

$(\mathrm{N}, \mathrm{N}, \mathrm{N}, \mathrm{N}$,$) and (-\mathrm{N},-\mathrm{N},-\mathrm{N},-\mathrm{N})$. This macroscopic state could have a dipolar (like the electronic orbital $2 \mathrm{p}$ ) or hyperspherically symmetric (like a $2 \mathrm{~s}$ electronic orbital). We believe that the likely topology is of a $2 \mathrm{~s}$ hyperspherical orbital with metric compression in the center and stretch on the outer layer. The decaying of this state, followed by recombination of the inner core would generate gamma rays that would propel the outer layer. The Quantum Lagrangian Principle (QLP) describing interacting precludes this explosion to be like anything we are familiar with. Quantum Lagrangian Principle together with retarded potential interaction (interaction along $45^{\circ}$ angle) impose equipartition of energy between Radial and Tangential degrees of freedom. In a four-dimensional non-compact spatial manifold that translates into the instantaneous acceleration of all matter (dilators) in the Universe to the speed of light in a tangential and radial directions. It also forces the thickness along $\mathrm{R}$ to be infinitesimal (or very small)

\section{Non-critical antropic argument}

Preceding the Big Bang were dimensional transitions. At the time of the Big Bang, the Universe was a 184 light-seconds radius metric fluctuation in a $4 \mathrm{D}$ spatial manifold. This radius is a rough estimate based on Black Hole density and the current mass in the Universe. The only information it carries is that the initial fluctuation wasn't microscopic. The Universe was born quite large already. This is the size when the transition from a Black Hole Universe expands to become a Neutron Star Universe which later decays into protons, electrons and antineutrinos. Propelling this expansion is the recombination process taking place within this shell. We speculate that this moment made the process entropically irreversible (Figure 8).

When the 4D macroscopic fluctuation arises and decays, a myriad dilators are created (matter and antimatter). The distribution of matter and antimatter follows a 2 s hyperspherical orbital distribution (4D space). Recombination occurs at the edge between matter and antimatter. This initial recombination is what propels the whole Universe outwards traveling at the speed of light. Here is where spin quantization and tunneling frequencies plays the most import role on the Universe. From our discussion of dilators and the stroboscopic Universe interaction can only exist at specific angles, so the acceleration happens coherently even if the interaction takes place on several dilator cycles.

When one considers a coherent process like spinning, the first question is about dephasing processes. Current Science only speaks about dephasing processes with respect to the projection of spin within our 3D Universe. There is not concept of dephasing of the spinning process itself. In our model for matter, the spinning dephasing processes are represented by neutrinos (transmutation notes or chords).

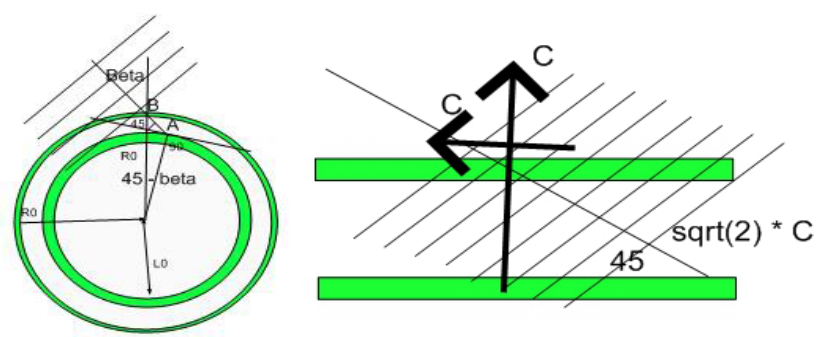

Figure 8: Time zero boundary conditions are shown above.

Imagine that the Big Bang created a distribution of radial velocities with a narrow peak at the correct equipartition (tangential kinetic energy=radial kinetic energy). Imagine that the process of blowing the external shell of the Initial Fluctuation was equivalent to blowing the Neutron-Star-like top layer of a Black Hole. Starting with neutrons is a sure way to end up with a neutral Universe and without any antimatter.

Let's consider neutrons that travelled at $1 \%$ faster than $c$ along the radial direction. By now, those neutrons would be protons and electrons and be at a hypersphere 0.1358 bly from us, frozen onto their FS (zero temperature) and have their stepwise expansion out of phase with ours. The Quantum Lagrangian Principle would make the phase difference between our two hyperspherical universes to be different than an integer number of de-Broglie steps since their acceleration angle was different from 45 degrees. This means that any Universe that is not at traveling at $c$ will not interact with us, will contain much less matter, be distant and for all purposes do not exist.

Since the larger the number of particle the sharper the distribution, one would expect that the largest fraction of the non-recombined matter is traveling with us.

This is a Non-Critical Anthropic argument, that is, it doesn't depend critically on anything. The only thing it depends is that there is a 4D spatial manifold and the interaction happens through 4D metric waves governed by a No-Work-by-Dilators Principle (Quantum Lagrangian Principle or Lazy Dilators Principle or Space Deformation Quantization). Spinning phase was also synced by the same logic.

\section{What is the essence of a universe?}

What makes a Universe is interaction! All laws of Physics depend upon that. To interact, particles should be within the same hypersphere and traveling at the speed of light (c). That condition allows for the working of retarded potentials, for our intermittent and yet continuous interaction. Caveat remains for the Lagging Hypersphere (LH) filled with Antimatter and moving less than a femtometer behind us. LH is the proposed source of Dark Matter within HU.

\section{Vacuum fluctuations}

We postulate that the spatial manifold is defect-free and that effectively there isn't anything ahead of us (energy equipartition at time zero demands a single Universe radial velocity c). Anything with radial velocity different from $\mathrm{c}$ will be too far behind or too far ahead of us to interact.

What about the paradigm of vacuum (zero-point) fluctuations? HU considers that quantum systems have zero-point fluctuations. Empty space, has no boundaries and thus no stationary states. This means that space itself doesn't have particles popping in and out of existence. This is a useful paradigm when one considers scattering process and center 
of mass kinetic energy is used to defined the range of states available for products.

This view eliminates the paradox associated with ultraviolet catastrophe.

\section{Photon}

HU Standard Model based upon the Fundamental Dilator implies that the whole Universe is permeated with metric waves or dilaton field. Those waves have very small wavelength ( 0.19 femtometer). They are generated as the dilator shape-shifts and spins within the $4 \mathrm{D}$ spatial manifold. Not surprisingly, this field is modulated by the location of the source (dilator). A photon is mapped to the position dependent spatial modulation of the dilaton field (Figure 9).

Since the amplitude of the dilaton field is quantized, photons are quantized twice. The first time it is because a photon is related to the dilaton field of a single dilator. The second time is due to them being derived from a transition within a quantized system. Since absorption requires a dephasing event and dilators are only located at the hypersphere, any absorption will only occur through the optical path where photons are effectivelly hypersuperficial waves. Unlike the underlying dilaton field (which never dephases), electromagnetic waves dephases and thus are effectively hypersuperficial waves.

\section{Asymptotic freedom}

Interaction occurs at 45 degrees through retarded potentials. This means that the Quantum Lagrangian Principle will lead to smaller and smaller accelerations as the local torsional angle approaches 45 degrees. Conversely, under extreme density (e.g. inside a Black Hole), there will be a density where the Force goes to zero again.

\section{HU and space twisting}

In HU, motion occurs to relax local space twisting (Figure 10).

\section{Hypergeometrical Standard Model}

\section{The fundamental dilator}

We propose that Fundamental Dilator Coherences (FDC) are the basic building block of matter. They are coherences between two

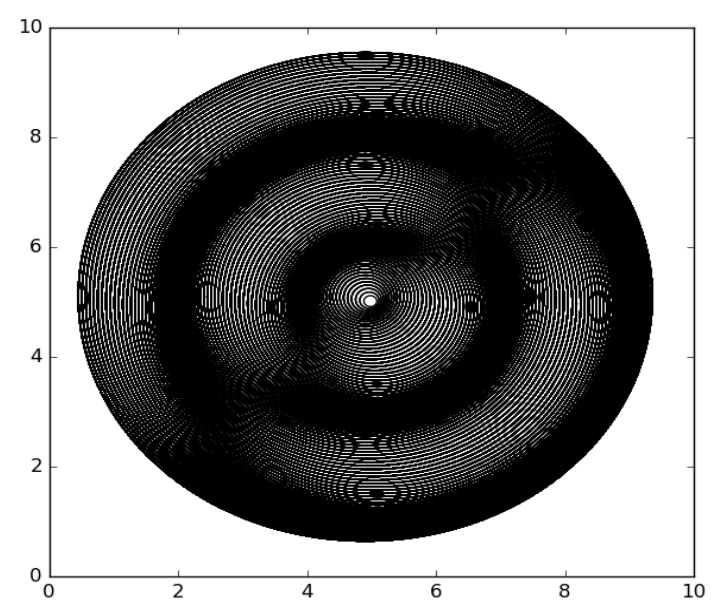

Figure 9: This figure shows a fine grid corresponding to the dilaton field and a much coarser modulation due to dipole oscillation.

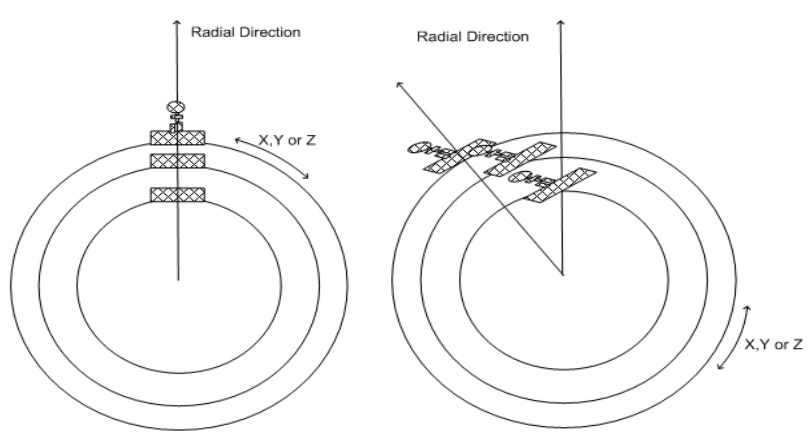

Figure 10: This figure shows the effect of a local twisting on the Fabric of Space (FS). Relaxed FS yields radial motion. Twisted FS results also on a tangential motion.

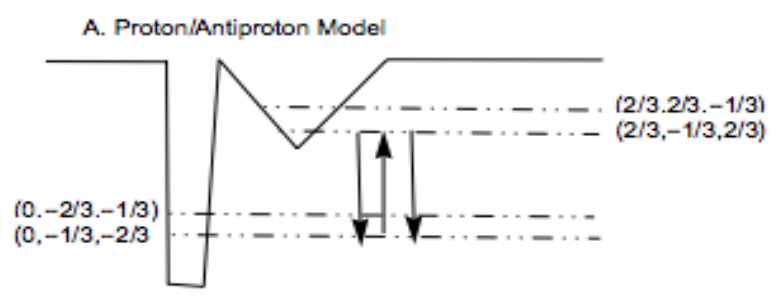

proton $\rightarrow$ positronRotated $\rightarrow$ antiproton $\rightarrow$ electronRotated

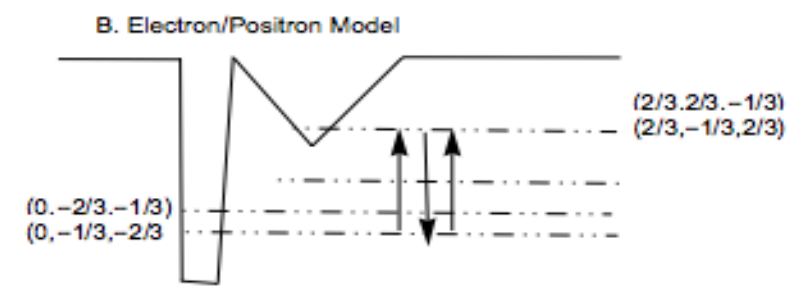

electron $\rightarrow>$ protonRotated $\rightarrow$ positron $\rightarrow$ antiprotonRotated

Figure 11: (a) Electron (Positron) and (b) Proton (antiproton) states.

metric deformation stationary states in a rotating four-dimensional double potential well. A single coherence between two $4 \mathrm{D}$-space deformation states or fundamental dilator is proposed to account of all the constituents of non-exotic matter (isotopes, neutrons, electrons and protons and their antimatter counterparties) and hyper-nuclei (hyperons) on Hypergeometrical Universe Standard Model Section. This coherence is between two deformation states with $4 \mathrm{D}$ volumes corresponding to the electron and proton, or electron-proton coherence. Here the proton, anti-proton, electron and positron are the same particle or the fundamental dilator, just four faces of the same coin (Figure 11).

Not represented in this graphic is the orientation of the state which can be in phase (flushed with the 3D Hyperspherical Universe) or offphase (perpendicular or rotated to the 3D Universe) nor the positive or negative (stretch or compression of space) phase information (Figure 12).

Half antineutrino transmutes an electron into a proton and vice-versa and it is named a transmutation chord. Similarly, from the assignment of pions, a half muon neutrino is a transmutation chord that converts matter to antimatter (electron into positron). By analogy, half Tau Neutrino will likely convert a proton into an antiproton (Figure 13). 
Vertical letters mean the that state is perpendicular to the $3 \mathrm{D}$ Universe (small cross-section for interaction). Upside down means antimatter state. Green(yellow) means positive(negative) charges or a local dilation(compression) of the metric. Compression or dilation is arbitrary since at each half de-Broglie step of the Hyperspherical Universe expansion, the phase changes 180 degrees. The return arrow indicates that this is a loop for the lifetime of the coherence. In the case of the fundamental dilator states, there is no other state to relax and the coherence lives forever (very long time).

The $4 \mathrm{D}$-mass is mapped to the $4 \mathrm{D}$ displacement volume of each one of those states. To understand what is a displacement volume, let's first consider a unit radius $3 \mathrm{D}$ sphere in a $3 \mathrm{D}$ spatial manifold. If space is stretched slightly along two axes and compressed along a third axis (e.g. $2 / 3,2 / 3,-1 / 3$ or the proton) by some very small arbitrary amount $(2 / 3,2 / 3,-1 / 3)^{*} A$, the change in volume can be approximated by the sum of those coefficients $(2 / 3+2 / 3-1 / 3)^{*} A=A$.

Due to the inherent anisotropy of an expanding hypersphere, tangential and radial states are modeled to have approximately a unit volume while differing by an anisotropic coefficient very close to unit.

\section{Inertial Mass=Dilator 3D Footprint!}

Since we know the masses of the 3D footprints electron and proton and since each one of these particles have positive and negative

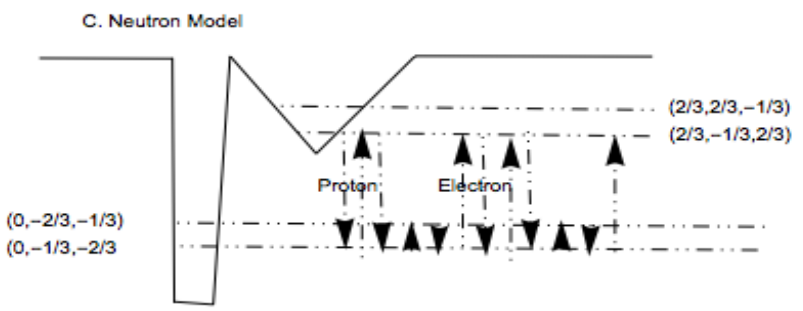

D. Electron Anti-Neutrino Model

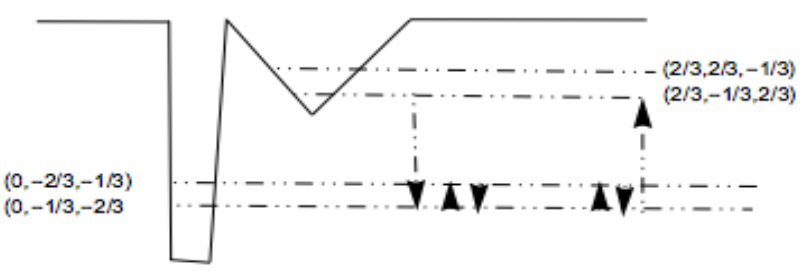

Composed of two half-antineutrinos

Figure 12: (c) Neutron, (d) Electron Anti-Neutrino composed of two transmutation chords (half antineutrino). footprints, the $4 \mathrm{D}$-mass of a Fundamental Dilator is considered to be the same as a Hydrogen Atom in atomic mass units to extract an isotropy.

The $3 \mathrm{D}$-mass is related to inertia, while the $4 \mathrm{D}$ mass is related to the particle ability to create dilaton waves (dilaton field). All four states have the same ability and thus the same absolute charge.

Up to now we described charged dilators. Let's now consider a neutron and a hydrogen atom.

\section{Neutron and the hydrogen atom}

The circular nature of the coherence above indicates its cyclic nature. This cycling will last the lifetime of the particle (Figures 14 and 15).

Each four balls correspond to the four states of the Fundamental Dilator Coherence. The red lines are transmutation chords (half electron-antineutrino) responsible for transmuting an electron into a proton at each de-Broglie cycle. In this model, one considers that the energy associated with tunneling could be momentarily converted into a 3D rotation while spinning continues. This means that the last state (electronRotated) in the proton sequence would remain in the electron state, execute a rotation around an axis in the 3D Universe while rotating $90^{\circ}$ perpendicular to $\mathrm{R}$. This would place the next state as being electron flush with the hyperspherical surface, thus transmuting from proton into an electron within the coherence (Figure 16).

The neutral nature of the neutron is the result of being a rotating dimer with zero net charge.

\section{Pions and antimatter}

Issues related to the total spin of a coherence depend upon the sum of the individual sub-coherence spins and that includes transmutations chords (half neutrinos). Pion Decay Channels are shown in Table 2 (Figures 17-19).

The muon neutrino can be detected at different Glutonic States where it performs one or more electron capture. The energy of the annihilation of the electron and proton is converted into kinetic energy for the decay products. Glutonic states are states that differ by the number of EFD internal coherences (e.g. Delta Plus Plus). Internal coherences are coherences not separated by a transmutation chord, thus they add no extra torsion to the composite coherence. They will be reviewed elsewhere.

The four fundamental particles (electron, proton, positron, antiproton) are modeled as different phases of the coherence between stationary deformation states of the local metric. The involved states express their nature (physical properties). Thus, at the first glance, the EFD should have a 4D-Mass identical a Hydrogen atom.
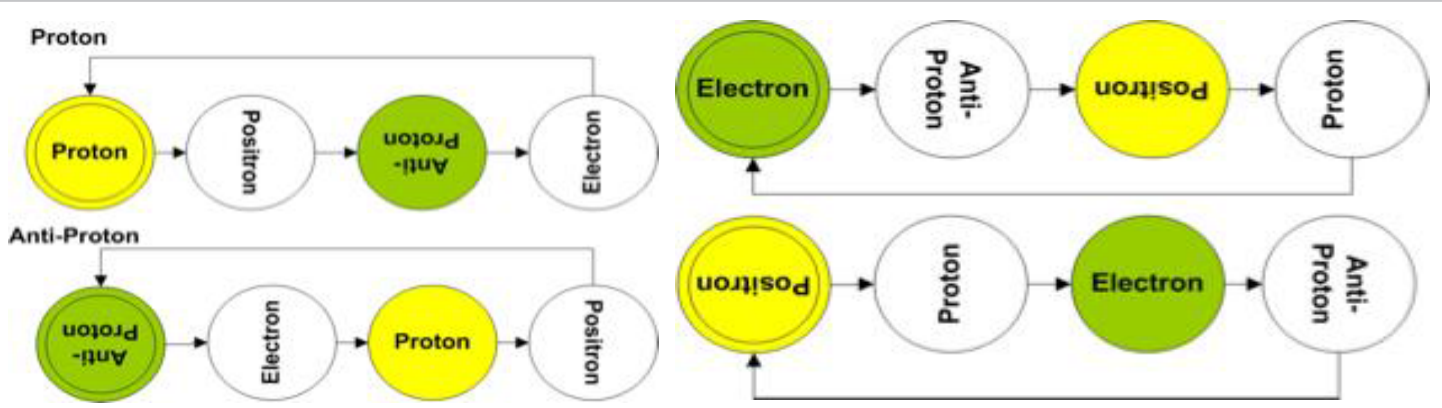

Figure 13: Diagrams representing the four fundamental particle coherences. 


\section{Hydrogen}

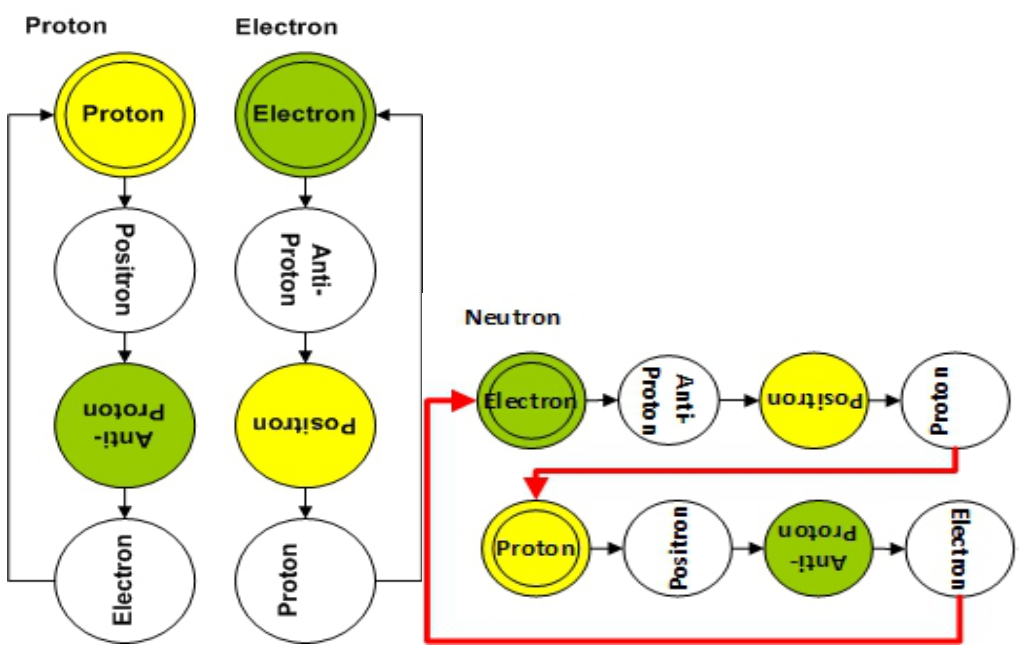

Figure 14: Hydrogen atom representing two interacting dilator coherences and neutron representing a composite coherence.

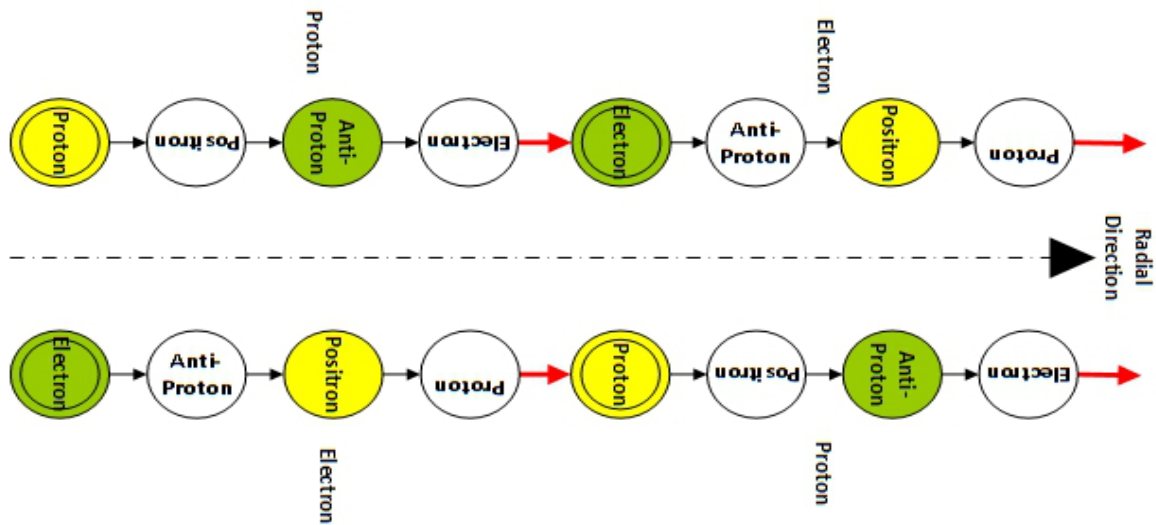

Figure 15: Neutron represented by a dimer, that is, two coherences composed each of two alternating chords, rotating $180^{\circ}$ at each de-Broglie step of the 3D Universe expansion.

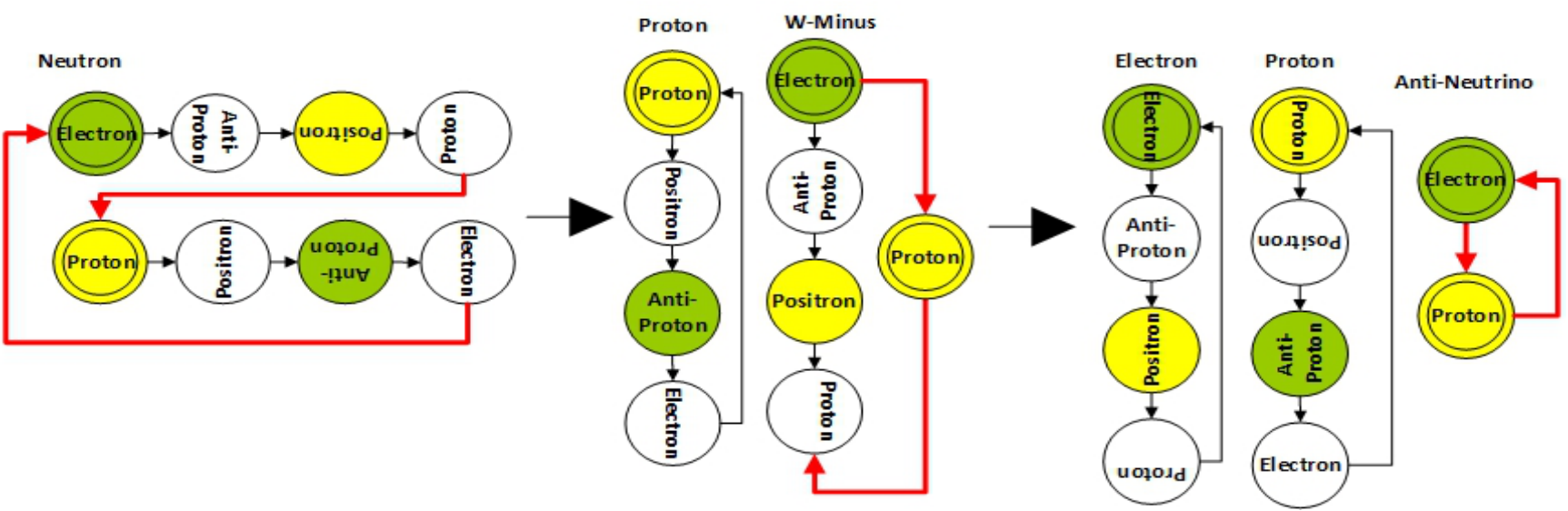

Figure 16: Neutron decay into W-Minus which subsequently decays into an electron and an electron anti-neutrino. 
Citation: Pereira MA (2016) The Hypergeometrical Universe: Cosmogenesis, Cosmology and Standard Model. J Generalized Lie Theory Appl 10:

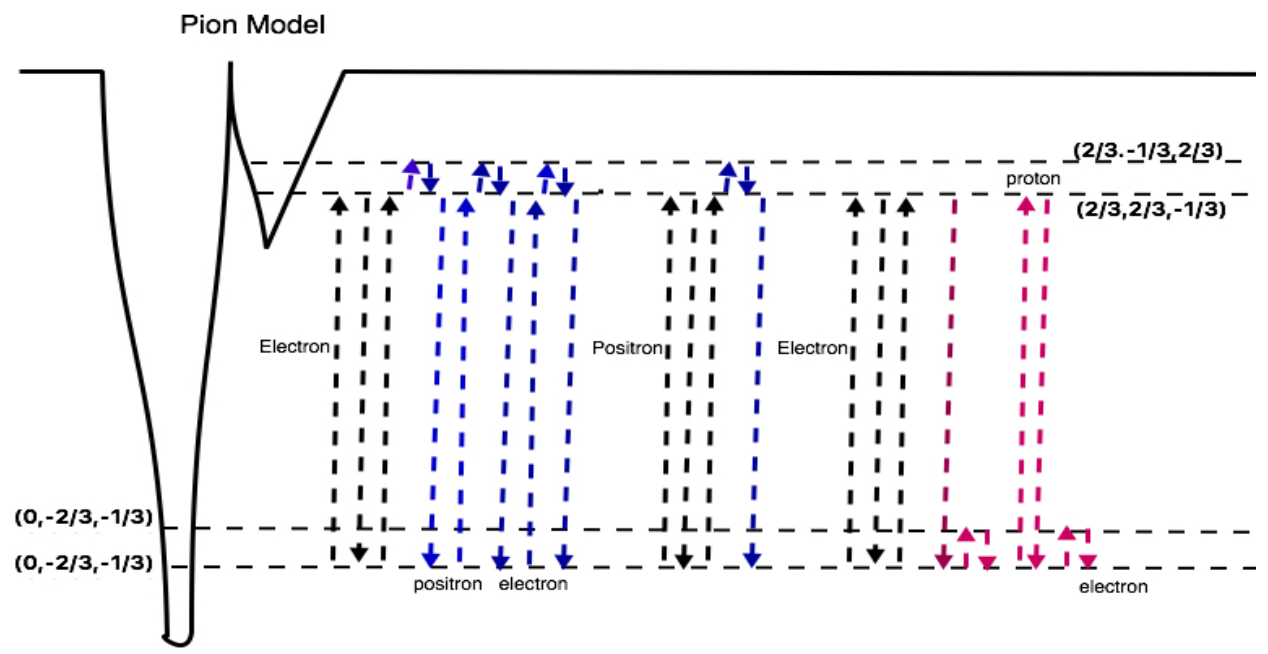

Figure 17: Pion Minus Diagram showcasing both transmutation chords.
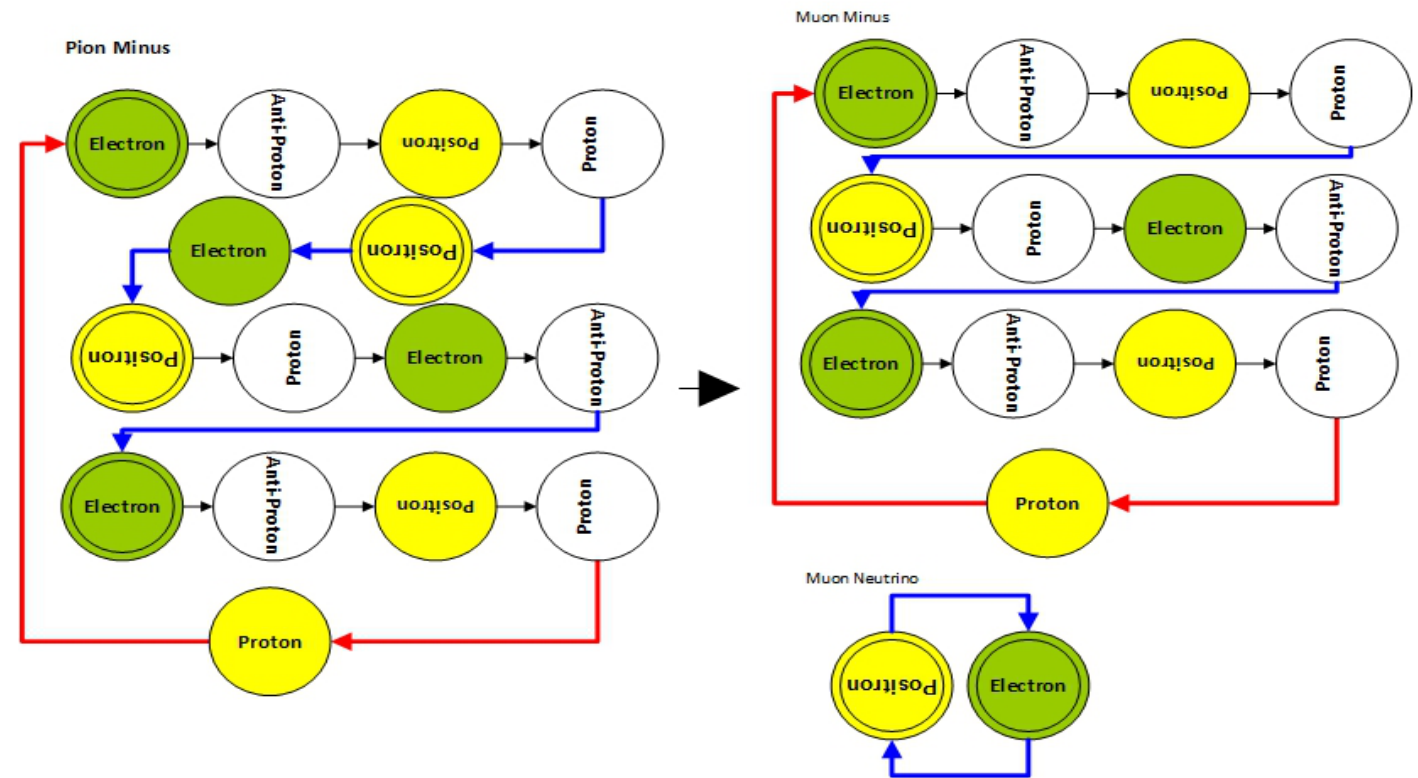

Figure 18: Pion Minus decay into Muon Minus and a Muon Neutrino.

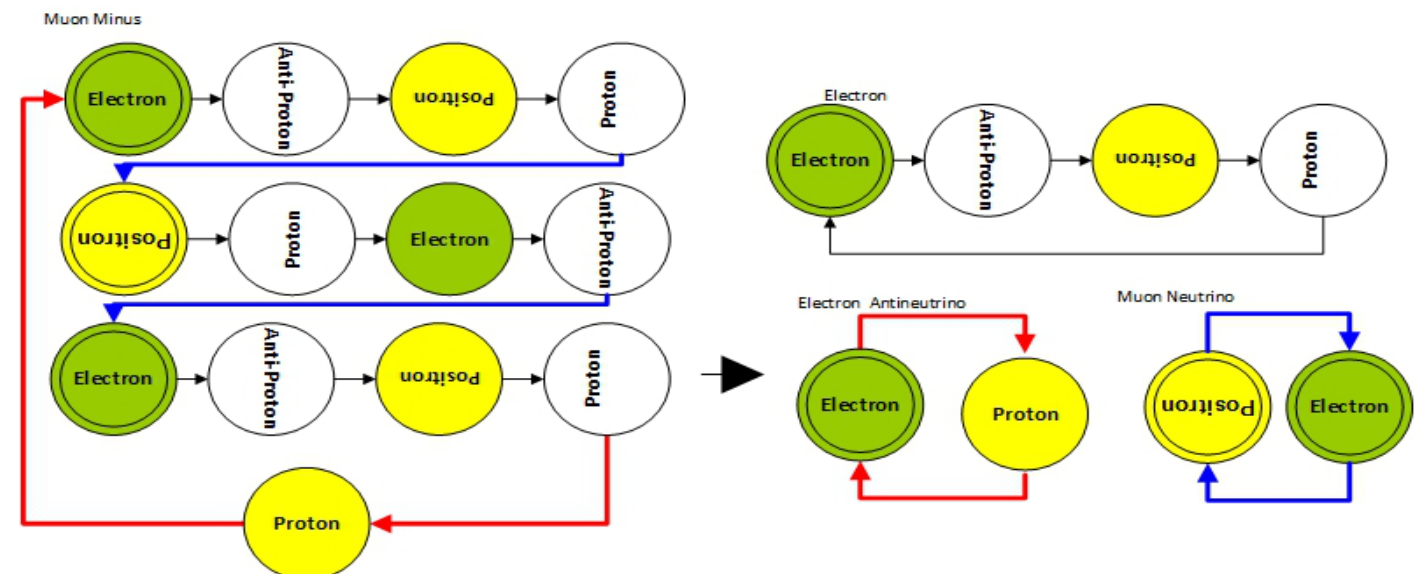

Figure 19: Muon Minus decay onto an electron and a muon neutrino. 


\begin{tabular}{|c|c|c|c|c|}
\hline Particle & Symbol & Mass & Decay Reaction & Spin \\
\hline Pion Minus & $\pi^{-}$ & 139.5702 & $e^{-}+n_{e}$ & 2-Jan \\
\hline Pion Minus & $\pi^{-}$ & 139.5702 & $m^{-}-n_{m}$ & 2-Jan \\
\hline Muon Minus & $m^{-}$ & 105.7 & $e^{-}-n_{e}+n_{m}$ & 2-Jan \\
\hline \multicolumn{5}{|c}{ Table 2: Pions. }
\end{tabular}

During the time taken to traverse a de-Broglie step, the dilator goes through its four phases (electron, proton, positron and antiproton) while spinning $360^{\circ}$ around an axis perpendicular to the direction $\mathrm{R}$ ( $\mathrm{R}$ is normal to our 3D Universe hypersurface). All times (proper time and Cosmological time $\Phi$ ) are made dimensional by the multiplication by the speed of light $\mathrm{c}$.

Pseudo-Time Quantization/de-Broglie Stepwise Expansion of the Universe are the result of the proposed model for matter based upon the Fundamental Dilator together with the proposed topology.

\section{Neutrinos ghostly nature}

From the diagrams, the frequency of tunneling for neutrinos is different from the tunneling frequency between the Fundamental Dilator Coherence states. Since all known stable matter in the Universe is made up of composite coherences based on the Fundamental Dilator, interaction will be limited by the time integral of the interaction. Only a very close collision will be effective. Anything else will result in no interaction.

\section{Particle topology}

Since the composite dilator coherences are degenerated (Figure 18) with respect the which state is initially in phase with the Universe, a trimer would have an equilateral geometry with respect to an axis perpendicular to the $3 \mathrm{D}$ Universe. Below is the topology of prime composite dilator coherences (hyperon family).

- Electron/Proton/Positron/Antiproton is a point

- Neutron is a line (segment)

- Pion minus/plus is a equilateral triangle

- Delta plus/minus are pentagons

- Kaons plus/minus are heptagons

- Xis plus/minus are undecagons

- Omega plus/minus are tridecagons

More complex hyperons will be presented elsewhere.

\section{Electromagnetic and gravitational dilators}

The archetypical Electromagnetic Dilator is represented by the Proton or Electron coherences presented previously. The Gravitational Dilator is represented by a spin zero Hydrogen Atom shown in Figure 20:

The first thing that comes to mind is that the Gravitational Fundamental Dilator contains two Electromagnetic Fundamental Dilators. Positive and negative phases of the dilator are positioned such as to minimize dilator work, that is, the phases are positioned to be in phase with the surrounding dilaton field.

Their 3D mass or inertial mass behaves as expected. An Electrostatic Fundamental Dilator on an electron pattern has the inertial mass of an electron. A Fundamental Dilator on a proton pattern has the inertial mass of a proton.

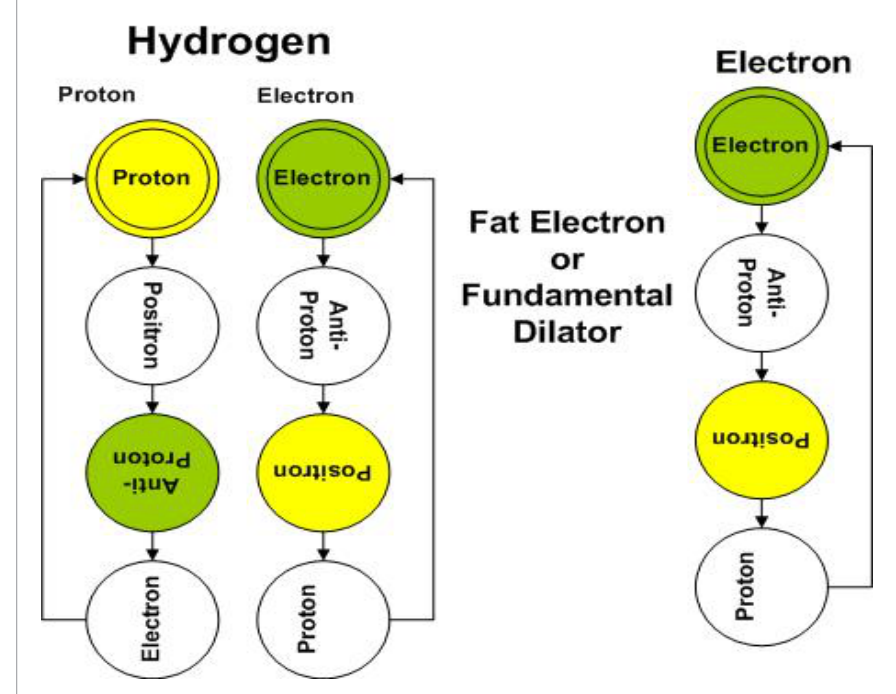

Figure 20: Archetypical Gravitational Fundamental Dilator (GFD=zero spin Hydrogen atom) and Electromagnetic Fundamental Dilator Coherence (EFD =electron).

\section{D de-Broglie waves and the de-Broglie force}

What are de-Broglie waves? Are they the same as the dilaton field?

Electron 3D de-Broglie waves have a wavelength that is different from the wavelength of a Fat Electron (our proposed view of the 4D displacement volume representation). The solution to the conundrum is that the dilaton tunneling, spinning and interaction with the $3 \mathrm{D}$ hypershell generates a bitonal dilaton field (two frequencies):

- one (our 3D de-Broglie waves) dependent upon the dilator footprint (3D-mass) on 3D Universe.

- one dependent upon the 4D Mass.

There is the question about their amplitudes, how similar are they? Just from inspection, they seen to be equivalent to a super strong gravity since they can be expressed by a small number of particles or even a single particle. Unlike gravity, uncorrelated particles or matter doesn't share the same wavelength as electromagnetism and gravitation. The de-Broglie dilaton field depends upon dilator footprint and velocity.

The answer to this question has implication on bunching and focusing of particles. This is extremely relevant to the pursuit of Coherent Nuclear Energy. The creation of coherent bunching of deuteron atoms for instance, followed by focusing and hadronic phase matching might be feasible.

When one focus particles, they would be subject to this bitonal dilaton field components. If the de-Broglie field is stronger than the EFD dilaton field, that might mean that homogeneous bunching with a larger number of particles, might be easier done than one with a small number of particles, clearing the path to coherent hadronics.

de-Broglie dilaton field might be bunching and debunching depending upon which phase you consider. Both phases define a trough that might neutralize enough electrostatic repulsion to allow for phase matched nuclear reactions.

First let's derive the de-Broglie law using the proposed $4 \mathrm{D}$ topology. Let's consider de-Broglie waves and the dilaton field for a hydrogen atom. In the case of a Hydrogen atom, the atomic mass is HydrogenMass. 
Let's consider a Hydrogen atom traveling at the speed of light along the 3D Universe. The vertical line points to the Radial direction (perpendicular to the $3 \mathrm{D}$ Universe). The line at $45^{\circ}$ corresponds to traveling at the speed of light both radially and tangentially. The oblique line indicates the projection onto the 3D Universe. Simple geometry tells you how the actual wave gets projected onto the $3 \mathrm{D}$ Universe (Figure 21).

The $45^{\circ}$ projection maps into the Compton wavelength. The horizontal lines represent steps of the 3D Universe. From the Figure 21 , it is clear that the de-Broglie wavelength in $3 \mathrm{D}$ is twice the one seen in $4 \mathrm{D}$. This creates a coincidence between the GFD dilaton field and the de-Broglie field.

The de-Broglie equations that calculate wavelength consistent with this $4 \mathrm{D}$ Perspective are given by:

$$
\begin{aligned}
& \lambda_{3 D}^{\text {deBroglie }}=\frac{h}{m_{3 D} v} \\
& \sin (\alpha)=\frac{\lambda_{4 D}^{\text {Vol }}(v)}{\lambda_{3 D}^{\text {deBroglie }}}=\tan (\alpha) \cos (\alpha)=\frac{\tan (\alpha)}{\sqrt{1+\tan ^{2}(\alpha)}} \\
& \tan (\alpha)=\frac{v}{c} \\
& \cos (\alpha)=\frac{\lambda_{4 D}^{\text {Vol }}(0)}{\lambda_{3 D}^{\text {deBroglie }}(v)}=\frac{1}{\sqrt{1+\tan ^{2}(\alpha)}} \\
& \lambda_{4 D}^{\text {deBroglie }}(v)=\lambda_{4 D}^{\text {Vol }}(0) \sqrt{1+\tan ^{2}(\alpha)}
\end{aligned}
$$

Hypersuperficial mode: Calculate $\lambda_{4 D}^{V o l}$ equivalent to the $\lambda_{3 D}^{\text {de-Broglie }}:$

$$
\begin{aligned}
& \lambda_{4 D}^{\text {Vol }}(v)=\lambda_{3 D}^{\text {deBroglie }}\left[\frac{\frac{v}{c}}{\sqrt{1+\left(\frac{v}{c}\right)^{2}}}\right]=\frac{h}{m_{3 D} v}\left[\frac{\frac{v}{c}}{\sqrt{1+\left(\frac{v}{c}\right)^{2}}}\right] \\
& \frac{f_{4 D}^{V o l}(v)}{c \sqrt{2}}=\frac{m_{3 D} v}{h}\left[\frac{\frac{v}{c}}{\sqrt{1+\left(\frac{v}{c}\right)^{2}}}\right]^{-1} \\
& f_{4 D}^{V o l}(v)=\frac{m_{3 D} c^{2} \sqrt{2}}{h} \sqrt{1+\left(\frac{v}{c}\right)^{2}} \\
& \left.\lambda_{4 D}^{\text {Vol }} \cong \frac{h}{\mathrm{~m}_{3 D} c}\right|_{v \ll<c}
\end{aligned}
$$

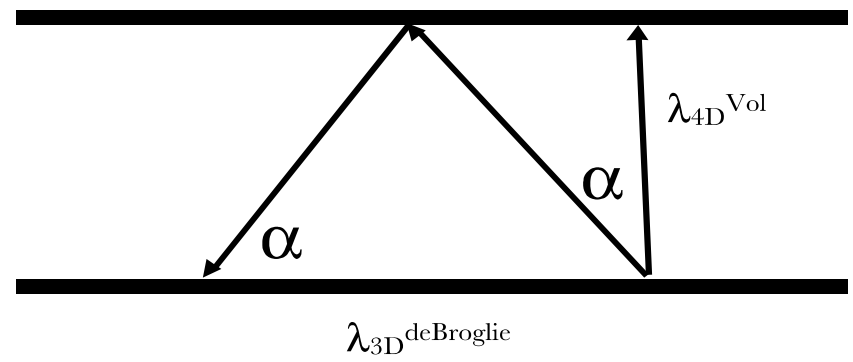

Figure 21: This diagram shows two consecutive de Broglie steps of the 3D Universe expansion and their relation to a volumetric dilaton wave. The $45^{\circ}$ projection maps into the Compton wavelength.

$$
\begin{aligned}
& f_{4 D}^{V o l}(v)=\left.\frac{2 m_{3 D} c^{2}}{h}\right|_{v=c} \\
& \lambda_{4 D}^{V o l}(v)=\left.\frac{h}{m_{3 D} v \sqrt{2}}\right|_{v=c}
\end{aligned}
$$

Hypervolumentric mode: Hypervolumetric modes anisotropy is derived the vacuum permittivity on equations $(78,86)$ :

$$
\begin{aligned}
& \lambda_{4 D}(v)=\frac{0.29273 h}{m_{4 D} c} \sqrt{1+\left(\frac{v}{c}\right)^{2}} \\
& f_{4 D}(v)=\frac{m_{4 D} c^{2} \sqrt{2}}{0.29273 h \sqrt{1+\left(\frac{v}{c}\right)^{2}}} \\
& f_{4 D}(c)=\frac{m_{4 D} c^{2}}{0.29273 h}=\frac{m_{4 D} c^{2}}{h^{G E}} \\
& h^{G E}=0.29273 h
\end{aligned}
$$

Equations 19-24. refers to the de-Broglie 3D waves and depends only on the $3 \mathrm{D}$ masses. It was made general to account for different velocities. The velocity is supposed to be invariant (low dispersion) between surface and volumetric dilatons. This is the equivalent hypervolumetric wavelength that is consistent with the observable $3 \mathrm{D}$ de-Broglie waves. One might conclude that the Electromagnetism and Gravitational dilaton field have $29.3 \%$ of the intensity of the de-Broglie dilaton field!

Equation (25-28) refers to the dilaton field created by all 4 phases of the dilaton coherence. One possible interpretation of the distinct nature of these dilaton modes is to map them to hypersuperficial (3D de-Broglie waves) and hypervolumetric (dilaton field responsible for volumetric forces electromagnetism and gyro-gravitation). There is an inherent uncertainty between superficial and volumetric waves in the context of waves on a surface attached to the moving frame of reference. The distinction should be made with respect to k-vectors. A superficial wave has a k-vector on the surface, while a volumetric wave has a k-vector perpendicularly to the surface that is free to move as the surface is tilted by interaction. Due to moving reference framework it is possible to map a superficial mode to a volumetric mode.

Again, the k-vector for these dilaton waves are perpendicular to the local FS. This is necessary for creating a simple picture of the 3D de-Broglie waves. The dilaton field propagating radially outwards corresponds to a dilator with zero velocity. Since the dilator k-vector is perpendicular to the $3 \mathrm{D}$ hypershell, the projection wavelength is infinite. As the dilator changes velocity, the reentering dilaton field projects the original wavelength into our known matter waves.

The Hypergeometrical Universe theory recognizes this as a yet unknown Force, not unlike Gravitation or Electromagnetism. The mathematical formulation for force calculation is identical to other forces and presented in the next section. Specifics of this force as well on how to physically mold spacetime will be covered in detail elsewhere.

\section{Hypergeometrical Universe Physics}

From Figure 1, the rate of torsion of the local FS is proportional to the force (Hypergeometrical Universe interpretation of Newton's Second Law is giving by:

$$
F=m_{03 D} c^{2} \frac{d \tanh \left(\alpha_{\tau}\right)}{d \tau}
$$


Adding the extra spatial dimension implies that:

$$
F=m_{03 D} c^{2} \frac{d \tanh \left(\alpha_{\tau}\right)}{d \tau}=m_{04 D} c^{2} \frac{d \tan \left(\alpha_{r}\right)}{d r}
$$

\section{Space stress-strain paradigm}

In a geometrical theory, the only relevant constructs are space, time, dilators, dilaton fields (dependent upon dilators position, velocity and space properties). A theory about the Universe based on those constructs would recast equation (30) as:

$$
\begin{aligned}
& \text { Stress }=\text { Area } 4 D \text { Strain } 4 D=\text { Area3DStrain } 3 D \\
& \text { Area } 4 D=m_{04 D} c^{2} \\
& \text { Area } 3 D=m_{03 D} c^{2} \\
& \text { Strain } 4 D=\frac{d \tan \left(\alpha_{r}\right)}{d r} \\
& \text { Strain } 3 D=\frac{d \tanh \left(\alpha_{\tau}\right)}{d \tau}
\end{aligned}
$$

The force between dilators can be calculated on the RXYZ frame.

From Figure 20, it becomes evident the reason why the 4D Mass of the Fundamental Dilator is initially mapped to the mass of a hydrogen atom in atomic units.

Transmutations chords redirect energy from tunneling into rotating in the $3 \mathrm{D}$ Universe, thus changing which phase is flush with the $3 \mathrm{D}$ Universe at the subsequent state. These chords (half-neutrinos) carry angular momentum since they correspond to rotations. They also carry linear momentum since they have a footprint on the Universe and are accelerated during the dissociation process. Since they have a different frequency, they will not produce anything that might be construed as a Gravitational nor Electromagnetic field. This would mean that it is meaningless the search for the neutrino mass as a potential indicator of the matter-induced spacetime curvature.

The EFD is a charged dilator and will be used as a probe for electromagnetism. For gravitation, the Fundamental Gravitation Dilator (GFD) archetype used will be a Hydrogen atom.

The introduction of a Fundamental Dilator and the concept of 4D Masses eliminates the asymmetry between electrons and protons and allow for the derivation of Natural Laws from first principles on a 5D Spacetime.

In this theory, a force capable of moving a body corresponds to a stress capable of deforming the Fabric of Space where that body is located. Notice that the body only has footprints on the FS where the dilators are. The strains are given by:

$$
\begin{aligned}
& \frac{d \tan \left(\alpha_{r}\right)}{d r} \\
& \text { and } \\
& \frac{d \tanh \left(\alpha_{\tau}\right)}{d \tau}
\end{aligned}
$$

where the angles are shown on the two cross-sections on Figure 1. The areas where the strain takes place are given by $m_{4 D}^{0} c^{2}$ and $m_{3 D}^{0} c^{2}$, respectively. They provide the extensive nature associated with mass in our current view.

Deformation of the Fabric of Space can be understood as acceleration from equation(30). Newton's Third Law also has a representation within this theory. The stress on interacting dilators (bodies) is also the same with opposing signs; this is equivalent to say that the force felt on each other is equal with opposite signs. This law is valid both on the RXYZ and in the $\Phi X Y Z$. In addition, one can equate

$$
\text { Area } 3 D(1) \operatorname{Strain} 3 D(1)=\operatorname{Area} 3 D(2) \operatorname{Strain} 3 D(2)
$$

where the indices refer to the particles. This applies to each de-Broglie step. This recast Newton's Third Law also as Archimedes Law of Lever if one focus on a single de-Broglie step.

Newton's fourth law is the Natural Law of Gravitation which will be derived later from first principles. The above equations are the basis for the more fundamental theoretical development in this theory. In first analysis, it is just an extrapolation of Newton's Law, which only covers the 3D space and introduces an unknown quantity $\mathrm{F}$ (Force). The introduction of a fourth spatial dimension allows for the creation of a purely geometric tautology relating Stresses on the two crosssections shown on Figure 1.

The stress associated with interaction is then same on both crosssections.

The strain is expressed differently in each cross-section and that permits the derivation of our fundamental laws of physics (Newton's, Gauss's, Biot-Savart's) from first principles.

Notice that the dilators will surf the XYZ on the XYZR crosssection. Our interpretation of events (dynamics) will be defined by the evolution of the dilator on $x y z \tau$ manifold.

\section{Pseudo time-quantization and the stroboscopic universe}

Pseudo Time-Quantization arises when one considers Newton's Law, where mass attracts mass at the direct products of their values. On the intermediate phases, the 3D overlap of the fundamental dilator with the FS goes to zero and so goes its perceived 3D mass, resulting in an intermittent interacting Universe (Stroboscopic Universe).

This pseudo-time quantization and the introduction of a fourthspatial dimension creates inherent uncertainties in the dynamics of dilator which together with the Quantum Lagrangian Principle would result in the basis for Quantum Mechanics. At each de-Broglie step, the next position where two interacting dilators (e.g. Hydrogen atom) would be depends upon their overlapping dilaton field at specific radial positions. The wavelengths and k-vectors on $X Y Z \tau$ depends upon velocity.

At any given step, the electron dilator should be in any one point on a circle drawn in the $3 \mathrm{D}$ space. That is the basis for the deterministic and yet uncertain motion in quantum mechanics. The loci of those steps should map to the probability density function. Since this theory is providing guidance for the underlying dynamics, it should be feasible to derive Schrödinger's equation from first principles.

In the past, I considered that the eigenstates would be stable in the sense of a Poincare' map, that is, one would start calculating trajectories:

1. start the trajectories from an initial position from the other particle (e.g. electron at position $\mathrm{x}$ from proton in a Hydrogen atom).

2. by using $3 \mathrm{D}$ interferograms (to calculate the forces actuating on the dilator at any given time).

3. use those forces to calculate motion on $X Y Z \tau$. Cycle back to item 2.

I expected that by starting the trajectories at different distances (different potential energies), stable Poincare' maps would naturally 
arise corresponding to the eigenstates. I considered that the de-Broglie field was present as an initial condition at each de-Broglie step of the Universe expansion.

After discovering the de-Broglie field (realizing that it was a real dilaton field mapped to a hypersuperficial dilaton mode), I realized that the de-Broglie field should be part of the protocol above to recover Quantum Mechanics. The reason being is the self-interaction with the de-Broglie field during the double slit experiment. If the field is there when there isn't any other potential, it should also be there when there is a potential.

This conclusion doesn't affect the derivation of Gauss, Newton and Biot-Savart Laws since they are large-body equations (non-Quantum Mechanical) that used the large $\mathrm{N}$ approximation in the derivation. Derivation of the Schrödinger equation or the Bohm equation is not in the scope of this work.

\section{Quantum lagrangian principle}

The Quantum Lagrangian Principle is nothing more than a direct result of the quantization of space deformation or metric deformation. It states that:

Dilators always dilate locally in phase with the surrounding dilaton field

Since Gravitation and everything else is described in terms of metric deformations, all fields are quantized in a sense but not in another. Gravitational/Electromagnetism fields are dependent upon dilaton fields from dilators which provide quantized dilations amplitudes and have to be at any given time on a well-defined spatial interference patterned grid, although not at quantized distances. This means that the generation of the field is quantized but the actual dilaton field is not.

This means that interacting dilators (e.g. Hydrogen atom composed of electron and proton), will always be at the nearest maximum dilation (contraction) for proton (electron) at each de-Broglie step of the Universe expansion. The phase choice is arbitrary. This means that the electron (the most mobile) will have an uncertain trajectory due to the azimuthally nature of the interferometric dilaton pattern resulting from proton-electron interaction.

The motion of a dilator can be thought in the RXYZ cross-section as being the interference between a self-wave which wavelength depends upon the torsional angle of the local metric. For relaxed space (angle=zero), the wavelength of a GFD is $\lambda_{1}$. If this dilator were accelerated to the speed of light tangentially (within the 3D), the wavelength would stretch to $\sqrt{2} \lambda_{1}$. This condition is required to keep in phase with the 3D Universe (Figure 22). $R_{0}$ is the age of the Universe

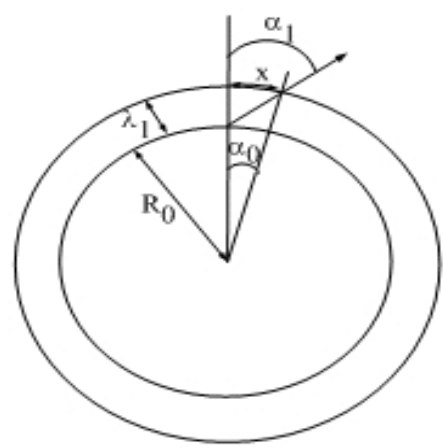

Figure 22: This figure shows a de-Broglie step $\lambda_{1}$ (Compton wavelength of a Hydrogen atom for GFD). times the speed of light. $\alpha_{1}\left(\alpha_{0}\right)$ corresponds to the FS normal direction for Electrostatic (Gravitational) interaction.

Due to the Quantum Lagrangian Principle, position $\mathrm{x}$ show in Figure 22 is calculated from the interference pattern between the dilator self-generated field and the Cosmological Field reaching that region of the $4 \mathrm{D}$ spatial manifold. The math is quite simple, just add the two waves and calculate the maximum or minimum. That will be the position of the dilator in the next de-Broglie step.

QLP applied to EFD allows the local metric deformation angle to be exactly the one calculated from $\mathrm{x}$ and $\lambda_{1}$.

QLP applied to GFD would have the same $\mathrm{x}$ motion but the FS deformation would be $\alpha_{0}$. Calculations indicate the existence of an adiabatic effect, thus yielding a larger deformation than expected.

Using the known Gravitational constant at $R_{0}$, a simple elasticity parameter is calculated. This allows for the calculation of the natural frequency of gravitational waves.

\section{Mechanism of attraction and repulsion}

To derive the laws of Nature, we first need a picture of what is happening during attraction or repulsion. We first write the deformational waveform for the dilaton field. This formula is valid for both electrostatic and gravitational interaction since the amplitude of each dilaton field is equal to the amplitude of the dilator at specific times of the expansion and they can be normalized to unit (Figure 23).

Mathematica Functions:

ladder $\left[x 1=\right.$ Heaviside Theta $[$ SawtoothWave $[x-0.25]-0.5]{ }^{*}$ Round $[A$ $b s[x]]$ pulses $[x 1=$ HeavisideTheta[SawtoothWave $[x-0.25]-0.5]$

The full dilaton field is given by

$$
\begin{aligned}
& \Psi_{1}\left(x, x_{0}\right)=\frac{\cos \left(k_{1} \cdot\left(x-x_{0}\right)\right)}{\left(1+f\left(k_{1} \cdot\left(x-x_{0}\right)\right)\right.} \\
& \Psi_{2}\left(x, x_{0}\right)=\frac{N \cos \left(k_{2} \cdot x\right)}{\left(1+f\left(k_{2} \cdot(R-x)\right)\right.} \\
& \Psi_{\text {Total }}\left(x, x_{0}\right)=\Psi_{1}\left(x, x_{0}\right)+\Psi_{2}\left(x, x_{0}\right) \\
& f(k x)=\text { ladder }[x]
\end{aligned}
$$

The function ladder is used to implement the dilution of the initial dilaton amplitude (unit) into the number of cycles. So the first cycle the intensity if 1 , at the second it is $1 / 2$, at the third it is $1 / 3$,etc. The only relevant part of the dilaton field for force derivation are the peaks because dilators land there at each step of the 3D Universe expansion. The derivative of the ladder function is zero for $\mathrm{x}=0$.

$$
\left.\frac{d f(x)}{d x}\right|_{x=0}=0
$$

The pulse function was used to show only that region and not the negative part of each cycle. Plots were generated with the function below:

$$
\Psi_{2}\left(x, x_{0}\right)=\frac{N \cdot \cos [k \cdot(R-x)]^{*} \text { pulse }[x]}{(1+\operatorname{ladder}(N \cdot(R-x)))} \approx \frac{N}{\left(1+\mathrm{f}\left(k_{2} \cdot(R-x)\right)\right)}
$$

if $\mathrm{N}$ (number of dilators) is very large, the oscillations are extremely close to each other and the approximate version of the equation (45) is used. Notice that when $\mathrm{x}-x_{0}=\mathrm{R}$ is a macroscopic distance (Figure 24): 


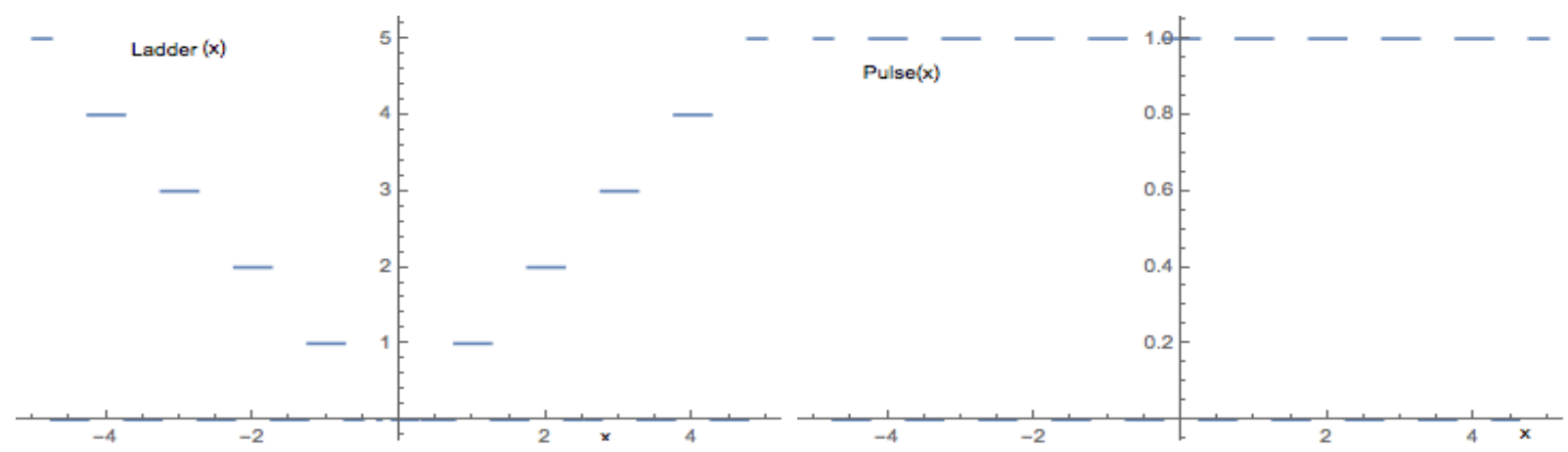

Figure 23: Ladder and pulse functions used to show how the dilator amplitude decays as a function of de-Broglie steps and cycles of the dilaton field.

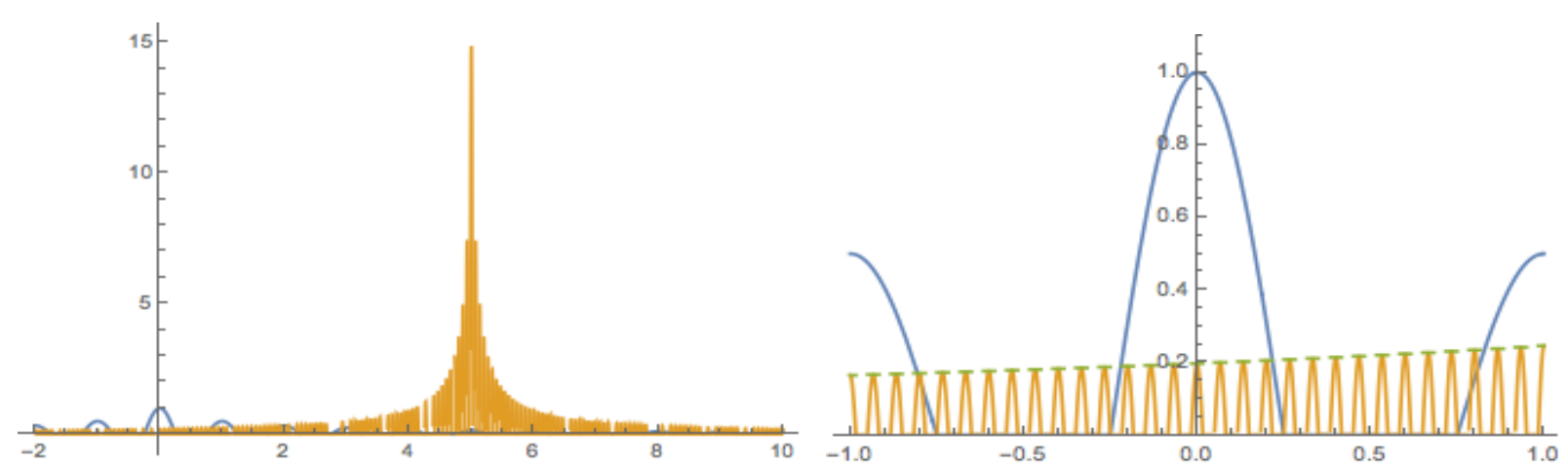

Figure 24: (a) shows a probe dilator interacting with a 4D Mass of 15 dilators. (b) shows where the dilator would land (slightly left from zero if this is an attractive interaction or to the right if not).

$$
\frac{d \Psi_{2}\left(x, x_{0}\right)}{d x}=\frac{N^{*} N^{*} k}{\left(N^{*} k^{*}(R-x)\right)^{2}}=\frac{1}{k N^{2}(R-x)^{2}}=\frac{N \lambda_{2}}{2 \pi R^{2}}
$$

Attractive or repulsive interaction are defined by the gradient of the peaks, which depends if the initial oscillation was positive or negative. In the case of Gravitation, the picture remains the same, but the amplitudes are much smaller due to countervailing actions of positive and negative dilators. The increased amplitude of the dilaton field with increasing number of dilator is the result of the Quantum Lagrangian Principle.

Dilators' amplitudes add together as opposed to averaging to zero in an ensemble. The increase in frequency is due to the physical volume from which the dilaton field emanates, thus arriving at different phases at any given point of space but adding together back properly at the right positions.

Attraction happens when interaction reduces local metric deformation. Repulsion happens otherwise.

For gravitation the picture is similar. The question is how the gradient is different for matter and antimatter? Van der Walls forces are always attractive and that might be the case for Gravity since they originate from a similar process.

This is a relevant result since it might answer the question about why don't we see antimatter. If antimatter produces antigravity, we might have clusters of antimatter galaxies, segregated by antigravity if not in this quadrant, perhaps in another quadrant of the Hyperspherical 3D Universe.

A more likely scenario is that all antimatter was left behind in the inner $2 s$ shell of the initial $2 s$ hyperspherical macroscopic metric fluctuation and that antimatter creates Gravity.

This theory provides a path for matter-antimatter conversion, thus opens the door for antimatter propulsion and gravity suspension. Other paths to gravity shielding will be discussed elsewhere.

\section{The meaning of spin}

The Figure 25 shows the formation of ortho- and para- Hydrogen atoms. Para-Hydrogen has lower energy due to the attractive interaction during the perpendicular phases. Similar considerations are applicable to Cooper Pairs in Superconductivity. In fact, in Cooper pairs, the intermediate matching is perfect. This understanding of spin will allow for the exploration of hidden Universe that is here when we momentarily don't exist (in the sense, I interact, therefore, I exist).

\section{The meaning of inertia}

Inertia maps to the overlap of the dilator with FS at specific phases when the Universe interacts. At those phases, the larger the overlap, the larger the inertia will be. The reason lies on the Stress-Strain view of interaction. Interacting dilators create dilaton fields which affect the position of other dilators at subsequent de-Broglie steps. This is equivalent to changing the propagation direction within the $4 \mathrm{D}$ spatial manifold and thus locally deform the FS. The larger the area that should be deformed the larger the required stress (Force), thus the larger the inertia.

The intersection of this $4 \mathrm{D}$ dilator displacement volume with the very thin $4 \mathrm{D}$ Universe (Fabric of Space) multiplied by a $4 \mathrm{D}$ mass density corresponds to the perceived 3D mass, a familiar concept. Since 


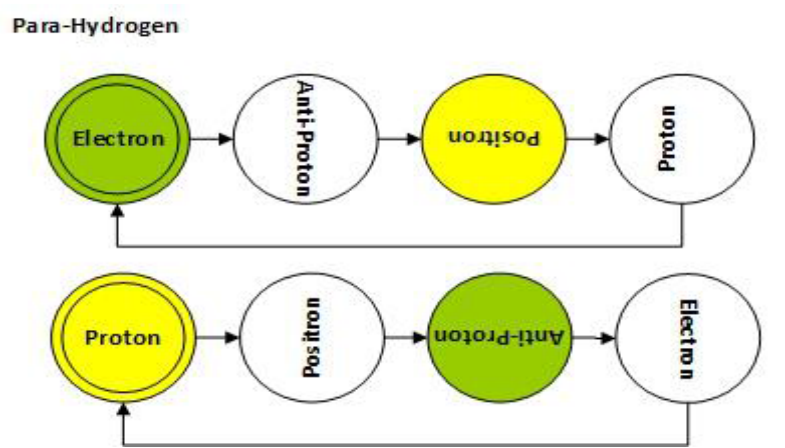

Ortho-Hydrogen

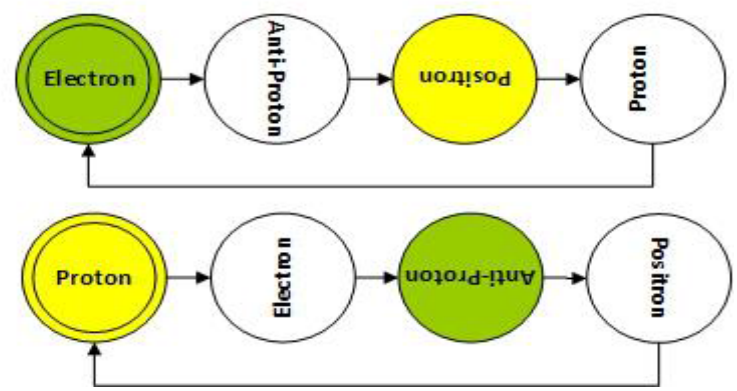

Figure 25: This figure shows that a negative spin means time reversing the cycle of the Fundamental Dilator Coherence.

both the dilator and the Fabric of Space are very thin, the intersection decreases extremely rapidly with spinning angle/phase tunneling. The interaction between dilators and dilaton fields (generated by other dilators) is directly dependent upon that footprint.

Since the footprint is non-null only at specific spinning angles, interaction is quantized and existence is quantized. Where existence was construed according to the following paradigm: I interact, therefore I exist. Neutrinos have been called Ghostly Particles due to their very small interaction with the rest of the Universe (dilators) and different de-Broglie wavelength. Figures 12 and 14 shows that neutrinos correspond to coherences with different wavelength or frequency than the Fundamental Dilator, thus resulting in alternating interactions that are only effective at very short range, thus making neutrino matter interaction cross-section very small.

\section{Newton's first law - Why do things keep moving?}

Eppur si muove: The local metric is perpendicular to the direction of propagation. It shows that the angle with respect to the radial line becomes smaller (more relaxed FS) as motion takes place and asymptotically the Cosmological angle tries to reach alphal or the initial local metric twist angle (Figure 26).

As time goes by, the local metric deformation described by the angle $\alpha_{1}$ becomes the smaller $\alpha_{2}$, thus motion results in a more relaxed local metric. Conversely, one could say that that is the reason for inertial motion, thus providing a reason for Newton's First Law. Alpha equal zero means that the dilator would propagate along the radial direction and that the local metric would be totally relaxed. This also means that current Universe should be mostly relaxed.

Notice that the apparent motion will still exist since the fabric of space is expanding and any place in the $3 \mathrm{D}$ universe has a Hubble expansion velocity. Although moving relatively to its original position, the body remains static with respect to the fabric of space (alpha parallel to R). At that point, the local deformation ceases to exist and the body drifts with the expansion at the Hubble velocity. In other words, motion is a way for $4 \mathrm{D}$ space to relax; in the same way, a tsunami is the means for the sea to regain a common level.

\section{Cosmological coherence}

Given that dilators obey the Quantum Lagrangian Principle, thus are never dephased by interactions, then it becomes clear that all dilators are in-phase throughout the Universe, creating a Cosmological Coherence.

The existence of macroscopic coherence is the underlying reason

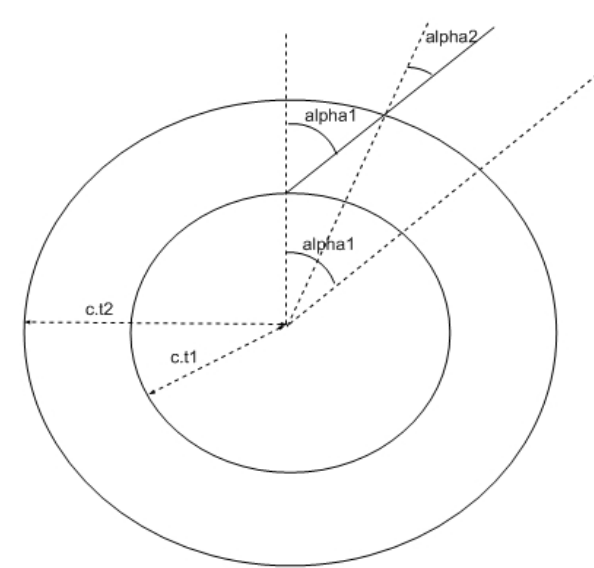

Figure 26: Here we present a dilator in motion and its position on two deBroglie steps.

why the concept of field can work. If one considers a field to be a property of space, then the coherent addition of dilaton fields is a requirement for the fields to be an extensive property of the number of dilators

\section{Dilator archetypes}

Before the derivation of Gauss Electrostatics and Newton's Gravitation Laws, lets discuss the meaning of a dilaton field for a Gravitational Fundamental Dilator (GFD) and for an Electromagnetic Fundamental Dilator. The electrostatic dilaton field is easy to understand

1. (EFD). Figure 20 shows the Electrostatic Fundamental Dilator going through the spinning and space deformational changes. Deforming space should create propagating deformation waves on the Fabric of Space. There is a subtle and very important difference between dilators and dilaton fields. Dilaton Fields propagate at the actual speed of light, $\sqrt{2} \mathrm{c}$. The 3D Universe propagates at $\mathrm{c}$ along the radial direction. The dilator is part of the $3 \mathrm{D}$ Universe and thus travels at that speed. Hence, a dilator is a dilaton wave generator that travels at lower speed that the waves it creates. At each de-Broglie step the local metric displacement volume is unit. In this theory, a unit amplitude of deformation is associated with the location where the dilator lands. This is the same for both electron state and proton state because they both have the same absolute value of coherently added $4 \mathrm{D}$ displacement volumes. 
2. (GFD) For the Gravitational Fundamental Dilator shown in Figure 20, the analysis is similar. Since GFD is a Hydrogen atom, any EFD (half of a GFD) will be both attracted and repelled. This process has similarity to Van der Walls forces. The difference is that the fluctuations are not related to charge position fluctuation but to charge transmutation at each half de-Broglie step, which happens in yoctoseconds.

Since the effect of these fluctuations on EFD comprising GFDs should be null, one would expect no effect force nor deformation of the local metric. If one starts with a relaxed local metric, the GFD should land on a place where the Fabric of Space is also relaxed. That is the support for the caption in Figure 22. Like in the Van der Walls forces, there will be a residual effect (Gravitation), that is, there will be an effective dilaton field generated and the corresponding GFD will follow the Quantum Lagrangian Principle and move to position $\mathrm{x}$ on Figure 22, independently of them being GFD or EFD. The difference will be what happens in subsequent steps. In the case of an Electrostatic Fundamental Dilator, the local metric will be twisted and the k-vector (direction where the EFD is traveling) will be changed by $\alpha_{1}$. In the case of a Gravitational Fundamental Dilator the change in k-vector is just $\alpha_{0}$.

To calculate the position $\mathrm{x}$ and thus the value of the Force, one needs to map charges and 3D masses to number of dilators. One $\mathrm{Kg} 4 \mathrm{D}$ of Hydrogen contains the same number of GFD dilators as one Kg4D of electrons (or protons) EFD.

\section{Relating charges and 4D masses}

First let's express Gauss law in terms of two interacting bodies of $\mathrm{N}=(1000$ Avogadro $) / \chi$ of dilators separated by one-meter distance. The reason for expressing Gauss Law in term of $\mathrm{N}$ is to have a term of comparison with Newton's Law, that is, both Gravitational and Electrostatic laws should be measuring the effect of the same number of dilators ( $\mathrm{N}$ electrons or $\mathrm{N}$ Hydrogen Atoms).

For the Electrostatic Force between two one N- EFD dilator masses:

The standard MKS equation for electrostatic force between two one Kg4D bodies of $\mathrm{N}=1000{ }^{*}$ Avogadro electrons ( $\chi$ a.m.u. electrons or protons) is giving by:

$$
\begin{aligned}
& F=\frac{1}{4 \pi \varepsilon_{0}}\left(\frac{\text { Coulombs }}{\text { meter }}\right)^{2}\left(\frac{E F D}{\text { Coulombs }}\right)^{2}\left(\frac{N E F D}{\chi K g 4 D}\right)^{2}\left(\frac{K g 4 D}{K g 3 D}\right)^{2}(K g 3 D)^{2} \\
& F=\mathrm{G}_{\text {Electrostatic }}^{4 D}\left(\frac{K g 3 D}{\text { meter }}\right)^{2}
\end{aligned}
$$

We make $((\mathrm{Kg} 4 \mathrm{D}) /(\mathrm{Kg} 3 \mathrm{D}))=1$ that is, we impose a one-toone mapping between a $3 \mathrm{D}$ volume (footprint of dilators in the $3 \mathrm{D}$ Hypersphere and the actual effective $4 \mathrm{D}$ volume). This is possible since we are using an effective 4D-Mass $\chi$ a.m.u. per dilator. This factor $\chi$ is justified in terms of anisotropy in and perpendicular to the FS.

$\mathrm{G}=$ gravitational constant $=6.6740810^{-11} \mathrm{~m}^{3} \mathrm{~kg}^{-1} \mathrm{~s}^{-2}$

$\chi$ is the EFD effective $4 \mathrm{D}$-Mass in a.m.u.

$$
\mathrm{G}_{\text {Electrostatic }}^{4 D}=\frac{1}{4 \pi \varepsilon_{0}(K g 3 D)^{2}} e^{2}\left(\frac{N}{\chi}\right)^{2}
$$

For the Gravitational Force between two one Kg4D dilator masses:

$F=G\left(\frac{1}{\text { meter }}\right)^{2}\left(\frac{\left(\frac{N}{2 \chi}\right) \mathrm{G} F D}{K g 4 D}\right)^{2}\left(\frac{K g 4 D}{K g 3 D}\right)^{2}(K g 3 D)^{2}=\mathrm{G}_{\text {Gravitational }}^{4 D}\left(\frac{K g 3 D}{\text { meter }}\right)^{2}$

$$
\mathrm{G}_{\text {Gravitational }}^{4 D}=G\left(\frac{1}{2 \chi}\right)^{2}
$$

So the ratio between the forces between two N-EFD (EGD) separated by 1 meter is given by:

$$
\frac{\mathrm{G}_{\text {Electrostatic }}^{4 D}}{\mathrm{G}_{\text {Gravitational }}^{4 D}}=\frac{1}{4 \pi \varepsilon_{0} G}(2 N e)^{2}=5 . E+30
$$

One $\mathrm{Kg} 4 \mathrm{D}$ of GFD contains half the number of EFD in the same displacement volume.

\section{Force unification}

Quantum gravity and electrostatic interaction:

Let's consider:

$$
\vec{r}_{0}=\left(\begin{array}{l}
0 \\
0 \\
0 \\
0
\end{array}\right) \text { and } \overrightarrow{\mathrm{R}}_{0}=\left(\begin{array}{l}
R \\
0 \\
0 \\
0
\end{array}\right)
$$

Vector expressed in terms of xyzR coordinates

After a de-Broglie cycle $\left(\lambda_{1}\right)$ :

$$
\vec{r}=\left(\begin{array}{l}
r \\
\lambda_{1}
\end{array}\right) \quad{ }_{0}=\left(\begin{array}{l}
R \\
0 \\
0 \\
\lambda_{1}
\end{array}\right) \vec{r}_{0}\left({ }_{1}\right)=\left(\begin{array}{l}
0 \\
0 \\
0 \\
\lambda_{1}
\end{array}\right)
$$

using director cosines $\alpha, \beta, \gamma$.

$\overrightarrow{r_{0}}\left(\lambda_{1}\right)$ is the unperturbed crest of our four-dimensional dilator 1 after a de-Broglie cycle.

$\vec{r}$ is the position of that same probe dilator under the influence of dilator 2 .

To analyze the interaction between a probe dilator and a $1 \mathrm{Kg} 4 \mathrm{D}$ body (N=1000*Avogadro and $1 \mathrm{Kg} 4 \mathrm{D}$ contains $\frac{N}{}$ EFD or GFD), let's express the dilaton field for a single particle as: $\chi$

$$
\Psi_{1}\left(x, x_{0}\right)=\frac{\cos \left(k_{1} \cdot x\right)}{\left(1+f\left(k_{1} \cdot\left(x-x_{0}\right)\right)\right)}
$$

Where

$$
f(\vec{k} \cdot \vec{r})=|\vec{k} \cdot \vec{r}|
$$

There is a constraint on $\mathrm{f}(\mathrm{x})$, which is that $\frac{d f}{d x}=0$ for $\mathrm{x}=0$. For any other position, $\frac{d f}{d x}=\vec{k} \cdot \vec{r}$.

For sake of plotting, we use a stepwise constant function, implemented by the function ladder $[\mathrm{x}]$.

Similarly, for a N-dilator body located at position $\vec{R}$ :

$$
\Psi_{2}\left(x, x_{0}\right)=\frac{N \cos \left(k_{2} \cdot(R-x)\right)}{\left(1+f\left(k_{2} \cdot(R-x)\right)\right)}
$$

where the effect of the $1 \mathrm{~N}$-dilator mass is implicit in the $\mathrm{k} 2$-vector and expressed by the factor $\mathrm{N}$. Later, when representing $1 \mathrm{Kg} 4 \mathrm{D}$ mass, we will replace $\mathrm{N}$ by $\mathrm{N} / \chi$. The wave intensity scales up with the number of particles $\mathrm{N}$.

$$
\left\|k_{2}\right\|=N\left\|k_{1}\right\|
$$

To calculate the effect of gravitational/electrostatic attraction, one 
needs to calculate the displacement on the dilaton field maximum around each particle or body due to interaction with the dilatons generated by the other body.

This is done for the lighter particle, by calculating the derivative of the waveform and considering the extremely fast varying gravitational wave from the macroscopic body always equal to one, since the maxima of these oscillations are too close to each other and can be considered a continuum.

The total waveform is given by:

$$
\Psi_{\text {Total }}\left(x, x_{0}\right)=\Psi_{1}\left(x, x_{0}\right)+\Psi_{2}\left(x, x_{0}\right)=\frac{\cos \left(k_{1} \cdot x\right)}{\left(1+f\left(k_{1} \cdot\left(x-x_{0}\right)\right)\right)}+\frac{N \cos \left(k_{2} \cdot x\right)}{\left(1+f\left(k_{2} \cdot(x-R)\right)\right)}
$$

$x_{0}$ is the position in the prior de-Broglie step of the Universe expansion.

\section{Why is the lightspeed $c$ the limiting speed in this Universe?}

The reason can be seen by taking of the derivative equation (42) with respect to $\mathrm{x}$ and equating it to zero. Normally we consider that $x_{0}=0$ as we are considering just the first step. Here we kept $x_{0}$ to determine asymptotic behavior of dilators under extreme fields.

Under extreme fields, let's consider that $\Phi_{2}$ is saturated, that is, it doesn't change with $\mathrm{x}$ anymore. Under those conditions, $\partial \Psi_{2} / \partial x=0$ and

$$
\frac{\partial \Psi_{\text {total }}}{\partial x}=\frac{\partial \Psi_{1}}{\partial x}=\frac{\left(k_{1} \cos \left[k_{1} x\right]\right)}{\left(1+f\left(k_{1}\left(x-x_{0}\right)\right)\right)^{2}}+\frac{\left(-k_{1} \sin \left[k_{1} x\right]\right)}{\left(1+f\left(k_{1}\left(x-x_{0}\right)\right)\right)}=0
$$

In the asymptotic regime, the next $\mathrm{x}$ will be equal to the prior $x_{0}$, thus the saturation angle will be such that:

$$
\begin{gathered}
\sin \left(k_{1} \cdot x\right)=\cos \left(k_{1} \cdot x\right) \\
\text { or } \\
k_{1} \cdot x=45^{\circ}
\end{gathered}
$$

A conclusion can be derived if one considers planar waves propagating along $\mathrm{R}$ and the position of the next $\mathrm{x}$ points to a $45^{\circ}$ from the $\mathrm{R}$ direction. This is the reason why the speed of light is the limiting speed when this paradigm is used for acceleration of masses.

That is also the reason why a Lorentz transformation and Strict Relative were created. Without the Quantum Lagrangian Principle and this proposed Universe Topology, the requirement of an accumulation point at $45^{\circ}$ can only be achieved using a hyperbolic XYZФ spacetime. The Hypergeometrical Universe Theory not only provides the reason why things move but also provides the reason why they cannot be accelerated faster than the speed of light. This also provides the basis for alternative understanding of the passage of time, space dilation etc.

Relating speed with the passage of time: Since at each de-Broglie step, the $\Delta \mathrm{x}$ associate with interaction depends upon the actual absolute speed (torsional of the local metric), when speeds are close to the speed of light, smaller $\Delta \mathrm{x}$ means slower dynamics (chemical or nuclear reaction dynamics). That in turn can be understood as slower passage of time. This means that, this theory states that the passage of time is constant. The laws of Physics are what needs change with speed.

This also means that a particle lifetime depends upon the torsion of its local metric. Nuclear energy is stored in internal rotational velocity, and thus on torsion of local metric. The effect of speed is to effectively relax the local metric, thus increasing the particle lifetime.

Deriving the Grand Unification Equation

To calculate the value of $\mathrm{x}$ in general, we consider that space is relaxed at time zero to make calculations easier and take the derivative of the dilaton field with respect to $\mathrm{x}$ in the proximity of the probe dilator $\left(x_{0}=0\right)$ and equate it to zero.

Notice that there is no need for any rescaling (Gravitation is much weaker than Electromagnetic interaction) or physical dimensions. Under those conditions:

$$
\begin{aligned}
& \Psi_{\text {Total }}(x)=\Psi_{1}(x)+\Psi_{2}(x)=\frac{\cos \left(k_{1} \cdot x\right)}{\left(1+f\left(k_{1} \cdot x\right)\right)}+\frac{N \cos \left(k_{2} \cdot x\right)}{\left(1+f\left(k_{2} \cdot(x-R)\right)\right)} \\
& \Psi_{\text {Total }}(x)=\frac{\cos \left(k_{1} \cdot x\right)}{\left(1+f\left(k_{1} \cdot x\right)\right)}+\frac{N}{\left(1+f\left(k_{2} \cdot(x-R)\right)\right)}
\end{aligned}
$$

For large $\mathrm{N}$

Taking the derivative at $\mathrm{x}=0$ :

$$
\begin{aligned}
& \frac{d \Psi_{\text {Total }}(x)}{d x}=-k_{1} \sin \left(k_{1} \cdot x\right)+\frac{N \cdot k_{2}}{\left(k_{2} \cdot R\right)^{2}}=0 \\
& k_{1}^{2} x=\frac{N}{k_{2} \cdot R^{2}} \\
& x=\frac{N \lambda_{1}^{2} \lambda_{2}}{(2 \pi)^{3} R^{2}}
\end{aligned}
$$

since

$$
\begin{aligned}
& \frac{d f\left(k_{1} \cdot x\right)}{d x}=0 \\
& \frac{d f\left(k_{2} \cdot(x-R)\right)}{d x}=k_{2} \\
& R>\lambda_{1}
\end{aligned}
$$

Where sine function was expanded into $k_{1} \cdot x$.

Let's define $\tan \left(\alpha_{1}\right)$ as:

$\tan \left(\alpha_{1}\right)=\frac{x}{\lambda_{1}} \delta=\frac{\left(\lambda_{1} \lambda_{2}\left(\frac{N}{\chi}\right)\right)}{(2 \pi)^{3} R^{2}} \delta$

$\delta$ is a parameter related to the type of interaction and the elasticity of space. Now we can calculate the acceleration as:

$$
\text { acceleration }=c^{2} \frac{d \tan \left(\alpha_{1}\right)}{d r}=\frac{c^{2} \tan \left(\alpha_{1}\right)}{\lambda_{1}}=\frac{c^{2} \lambda_{2}\left(\frac{N}{\chi}\right)}{(2 \pi)^{3} R^{2}} \delta
$$

Now we can calculate the electrostatic force between two $1 \mathrm{Kg} 3 \mathrm{D}$ mass of EFD with $\mathrm{R}=1$ meter. $(\mathrm{N} / \chi)=$ Number of EFD per $1 \mathrm{Kg} 4 \mathrm{D}$.

Remember that we are using a mapping

$$
\left(\frac{K g 4 D}{K g 3 D}\right)=1
$$

By allowing the actual 4DMass of a dilator to be given by $\chi$ a.m.u..

$$
\begin{aligned}
& F_{\text {Electrostatic }}=1 K g 3 D^{*} \text { acceleration }=\frac{c^{2} \lambda_{2}\left(\frac{N}{\chi}\right)^{2}}{(2 \pi)^{3}} \delta\left(\frac{1}{K g 3 D}\right)\left(\frac{K g 3 D}{\text { meter }}\right)^{2} \\
& F_{\text {Electrostatic }}=\mathrm{G}_{\text {Electrostatic }}^{4 D}\left(\frac{K g 3 D}{\text { meter }}\right)^{2}
\end{aligned}
$$




$$
\mathrm{G}_{\text {Electrostatic }}^{4 D}=\frac{c^{2} \lambda_{2}\left(\frac{N}{\chi}\right)^{2}}{(2 \pi)^{3}} \delta\left(\frac{1}{K g 3 D}\right)
$$

Comparing $G_{\text {Electrostatic }}^{4 D}$ with the previous calculated value using Gauss' Law:

$$
\frac{1}{4 \pi \varepsilon_{0}} e^{2}\left(\frac{N}{\chi}\right)^{2} \frac{1}{(K g 3 D)^{2}}=\frac{c^{2} \lambda_{2}\left(\frac{N}{\chi}\right)^{2}}{(2 \pi)^{3}} \delta\left(\frac{1}{K g 3 D}\right)
$$

For electrostatics, we will assign $\delta=1$, that is, the local surface is totally twisted by the dilaton field. Solving the equation for $\lambda_{2}$ :

$$
\lambda_{2}=\frac{2 \pi^{2} e^{2}}{\varepsilon_{0} c^{2}(K g 3 D)}=6.36737 * 10^{-43}
$$

$\lambda_{2}$ is the Compton wavelength of $1000^{\star}$ Avogadro of $14 \mathrm{D}$ a.m.u dilator. Subsequently, we will hide the Kg3D unit for convenience, but that unit is necessary to recover $\varepsilon_{0}$ proper units.

For the case of a 4DMass of 1 a.m.u., one can calculate the effective electron 4DMass :

$$
\frac{\lambda_{2}}{\frac{\left.\lambda_{1} \text { (a.m.u. }\right)}{1000 * \text { Avogrado }}}=\frac{\left(\frac{1}{\chi}\right)}{1}
$$

$\lambda_{1}=\frac{h}{\text { ca.m.u. }}=1.3205$ femtometer

$\chi=\frac{\lambda_{1}}{\lambda_{2} * 1000 * \text { Avogrado }}=1.70918$ a.m.u.

This result is the reasoning behind equation (18):

$$
\begin{aligned}
& \lambda_{14 D}^{E F D}=\frac{h}{c \chi\left(\frac{\mathrm{m}_{4 D}}{\text { HydrogenMass }}\right)}=\left(\frac{\text { HydrogenMass }}{\chi}\right) \frac{h}{c \mathrm{~m}_{4 D}}= \\
& =\frac{0.292731 h}{c \mathrm{~m}_{4 D}}=\frac{h^{G E}}{c \mathrm{~m}_{4 D}}=3.83507 * 10^{-16} \text { meters } \\
& h^{G E}=0.292731 h
\end{aligned}
$$

where $\mathrm{m} 4 \mathrm{D}=$ HydrogenMass for a EFD (electron or proton). $h^{G E}$ is the effective Planck's Constant for Hypervolumetric Dilaton Waves (see equation 18). Since the dilaton field for Gravitation is not the same as the $3 \mathrm{D}$ de-Broglie matter wavelength, one might consider using equation (18) for both EFD and GFD, thus

$$
\frac{\lambda_{14 D}^{E F D}}{\lambda_{14 D}^{G F D}}=2
$$

de-Broglie step characterization: To calculate the time for GFD (us and the whole Universe) to traverse a de-Broglie step: $\lambda_{1}=0.292731 \mathrm{~h} /$ (m.c) $=1.91753^{\star} 10^{-16}$ meters where

$$
\mathrm{m}=2 \text { HydrogenMass }
$$$$
t_{1}=\lambda_{1} / c=6.39621^{\star} 10^{-25} \text { seconds }
$$

is the time to traverse a de-Broglie step of $\lambda_{1}=0.191753$ femtometers

Effective 4D masses: Let's write our expression for the vacuum permittivity $\varepsilon_{0}$ : $\varepsilon_{0}=\frac{2 \pi^{2} e^{2}}{\lambda_{2} c^{2}(K g 3 D)}$

Let's define $\delta$ such that the angle is measure as related to Gravitation: $\delta=\frac{\lambda_{1}}{R_{0}} \xi$

Next we will explore a scenario where the space elasticity is considered constant throughout the Universe life. Let's analyze $\delta$ for Gravitation for $1 \mathrm{Kg} 4 \mathrm{D}$ of dilators.

$$
\begin{aligned}
& F_{\text {Gravitational }}=G\left(\frac{1}{2 \chi}\right)^{2}=\frac{\left(c^{2} \lambda_{2} M\left(\frac{N}{2 \chi}\right)^{2}\right)}{(2 \pi)^{3}} \frac{\lambda_{1}}{R_{0}} \xi \\
& \xi=\frac{(2 \pi)^{3} G R_{0}}{c^{2} \lambda_{2} N^{2} \lambda_{1}}=283,087
\end{aligned}
$$

$\mathrm{G}$ can be written as:

$$
G(R)=\frac{c^{2} \lambda_{2} N^{2}}{(2 \pi)^{3}} \frac{\lambda_{1}}{R} \xi
$$

Let's calculate the gravitational force acting on a mass $\mathrm{m}$ :

$$
F=m a=m c^{2} \frac{\partial \tan \left(\alpha_{0}\right)}{\partial \lambda_{1}}=\left(m c^{2}\right) \frac{\delta x}{\lambda_{1}^{2}}=m \frac{c^{2} \delta}{\lambda_{1}^{2}} x=m(2 \pi f)^{2} x
$$

Thus, the natural frequency of gravitational waves is:

$$
f=\frac{1}{2 \pi} \sqrt{\frac{c^{2} \delta}{\lambda_{1}^{2}}}=\frac{c^{2}}{2 \pi \lambda_{1}} \sqrt{\frac{\lambda_{1} \xi}{R_{0}}}=111,101 \mathrm{~Hz}
$$

Notice that this is not dependent upon any masses, only dependent upon the assumption of constant space elasticity. That should be the best frequency to look for or to create gravitational waves. Of course, Hubble red shift considerations should be used to determine the precise frequency from a specific region of the universe. The complete Gravitatostatic equation is given by:

$$
F_{\text {Gravitational }}=\left\{\frac{c^{2} \lambda_{2}\left(\frac{N}{\chi}\right)^{2}}{(2 \pi)^{3}} \frac{\lambda_{1}}{R_{0}} \xi\right\} \frac{(m 1 * m 2)}{R^{2}}
$$

Later we will derive a Gyrogravitational version of this equation.

Grand unification supersymmetry: As the dimensional age of the universe becomes smaller, the relative strength of gravitation interaction increases. Conversely, one expects that as the universe expands gravity will become weaker and weaker. This and the fourdimensional light speed expanding hyperspherical universe topology explain the acceleration of expansion without the need of antigravitational dark matter.

We can now calculate the radius of the Universe when Gravitational and Electrostatic forces were equal. Just make $=1$ and calculate $R_{0}$ :

$$
\begin{aligned}
& \delta=\frac{\lambda_{1}}{R_{0}} \xi \\
& R_{0}=\xi \lambda_{1}=283,087 \lambda_{1}=1.08566 * 10^{-10}=1.08566 \text { Angstroms } \\
& t_{0}=\frac{R_{0}}{c}=3.62137 * 10^{-19} \text { seconds }
\end{aligned}
$$

Thus, when $R_{0}$ was smaller than 283,087 times $\lambda_{1}$ (at $3.6 \mathrm{E}-19 \mathrm{~s}$ into the Universe life), gravitational and electromagnetic interactions had equal strength. They were certainly indistinguishable when the radius of 
the universe was one de-Broglie wavelength long. This section is called Grand unification supersymmetry, because condition in equation (57) plays the role of the envisioned group theoretical supersymmetry of the grand unification force. Of course, it has a geometrical interpretation. At that exact radius, an elastic spring constant of the fabric of space allows for a change in the local normal such that it becomes parallel to the redirection of k-vector of a freely moving dilator.

Quantum gravity: Quantum aspects can be recovered by not using fast oscillation approximation. It is also important to notice that equations (44) and (45) can be used to calculate the interaction between any particles (matter or anti-matter) or to perform quantum mechanical calculations in a manner similar to molecular dynamic simulations. The quantum character is implicit in the de-Broglie wavelength stepwise quantization. It is also relativistic as it will become clear when one analyzes magnetism next.

Magnetic interaction: The derivation of the Biot-Savart law: Let's consider two wires with currents $i_{1}$ and $i_{2}$ separated by a distance R. Let's consider $i_{2}$ on the element of length $d l_{2}$ as the result of a moving charge of mass of $1 \mathrm{Kg} 4 \mathrm{D}$ of electromagnetic fundamental dilators. This is done to obtain the correct scaling factor.

Without loss of generality, let's consider that the distance between the two elements of current is given by:

$$
r_{0}=\left[\begin{array}{l}
0 \\
0 \\
0 \\
R
\end{array}\right] \text { and } R=\frac{R}{\sqrt{3}}\left[\begin{array}{l}
1 \\
1 \\
1 \\
0
\end{array}\right] \text { and } \hat{R}=\frac{1}{\sqrt{3}}\left[\begin{array}{l}
1 \\
1 \\
1 \\
0
\end{array}\right]
$$

The velocities are:

$$
V_{1}=\left[\begin{array}{cc}
\dot{q}_{1} & 1 \\
v_{1} \beta_{1} \\
v_{1} \gamma_{1} \\
c
\end{array}\right] \text { and } V_{2}=\left[\begin{array}{c}
\dot{q}_{2} \\
v_{2} \beta_{2} \\
v_{2} \gamma_{2} \\
c
\end{array}\right]
$$

Since one expects that the motion of particle 2 will produce a drag on the particle 1 along particle 2 direction of motion. Particle 1 is located at position $R\left(\begin{array}{llll}0 & 0 & 0 & 1\end{array}\right)$, just prior to the next de-Broglie Universe expansion step. Particle 2 was placed at position $\frac{R}{\sqrt{3}}\left(\begin{array}{llll}1 & 1 & 1 & 0\end{array}\right)$ and traveling at speed $V_{2}=\left(\begin{array}{lllll}v_{2} & \alpha_{2} & v_{2} \beta_{2} & v_{2} & \gamma_{2}\end{array}\right)$. The retarded potential time difference is $\frac{R}{c}=\frac{\sqrt{2} R}{\sqrt{2} c}$ just to remind us that the actual light speed is $\sqrt{2} R$ and it traverses $\sqrt{2} R$ in a $4 \mathrm{D}$ spatial manifold at 45 degrees. The direction of the drag will be:

$$
\hat{r}+\frac{\vec{v}_{2}}{c}=\frac{1}{\text { NormalizationFactor }}\left(\begin{array}{c}
\frac{1}{\sqrt{3}} \\
\frac{1}{\sqrt{3}} \\
\frac{1}{\sqrt{3}} \\
-1
\end{array}\right)+\left(\begin{array}{c}
\frac{v_{2}}{c} \alpha_{2} \\
\frac{v_{2}}{c} \beta_{2} \\
\frac{v_{2}}{c} \gamma_{2} \\
1
\end{array}\right)
$$

The force will depend upon the distance defined by $\frac{R}{\sqrt{3}}\left(\begin{array}{llll}1 & 1 & 1 & 0\end{array}\right)$. The drag projection on reference frame of particle 1 , onto that vector should be made unit such that one can properly evaluate the force modulus. The Normalization Factor (NFactor) is given by:

$$
\begin{aligned}
& \text { NFactor }=\left[\begin{array}{llll}
\frac{1}{\sqrt{3}} & \frac{1}{\sqrt{3}} & \frac{1}{\sqrt{3}} & 1
\end{array}\right]\left[\begin{array}{cccc}
1 & 0 & 0 & -\frac{v_{1}}{c} \alpha_{1} \\
0 & 1 & 0 & -\frac{v_{1}}{c} \beta_{1} \\
0 & 0 & 1 & -\frac{v_{1}}{c} \gamma_{1} \\
\frac{v_{1}}{c} \alpha_{1} & \frac{v_{1}}{c} \beta_{1} & \frac{v_{1}}{c} \gamma_{1} & 1
\end{array}\right]\left(\begin{array}{c}
\frac{1}{\sqrt{3}}+\frac{v_{2}}{c} \alpha_{2} \\
\frac{1}{\sqrt{3}}+\frac{v_{2}}{c} \beta_{2} \\
\frac{1}{\sqrt{3}}+\frac{v_{2}}{c} \gamma_{2} \\
0
\end{array}\right)
\end{aligned}
$$

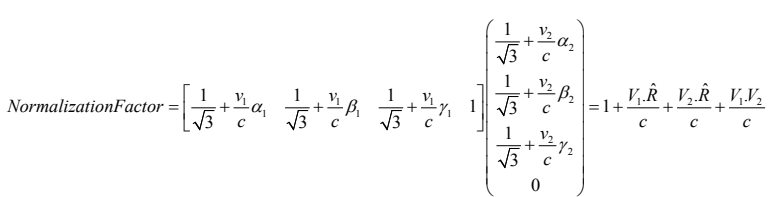

$$
\begin{aligned}
& \text { NFactor }=1+\frac{V_{1} \cdot \hat{R}}{c}+\frac{V_{2} \cdot \hat{R}}{c}+\frac{V_{1} \cdot V_{2}}{c^{2}}
\end{aligned}
$$

This normalization factor will be deployed at the end of the calculation. If it is deployed at the beginning, there wouldn't be any change in phase due to interaction.

Simple geometry (Figure 27) in $4 \mathrm{D}$ reveals that:

$$
\begin{aligned}
& \alpha=\operatorname{atan}\left(\frac{v_{2} \alpha_{2}\left(\frac{R}{c}\right)}{R}\right)=\operatorname{atan}\left(\frac{v_{2} \alpha_{2}}{c}\right) \\
& \cos \left(\alpha^{\prime}\right)=\cos \left(-\frac{\pi}{4}+\alpha\right)=\cos \left(\frac{\pi}{4}\right) \cos (\alpha)+\sin (\alpha) \sin \left(\frac{\pi}{4}\right) \\
& \cos \left(\alpha^{\prime}\right)=\frac{\sqrt{2} \cos (\alpha)}{2}[1+\tan (\alpha)] \\
& \cos \left(\alpha^{\prime}\right) \propto 1+\frac{v_{2} \alpha_{2}}{c}
\end{aligned}
$$

The last part of the identity above shows the position where Particle would be (referred from Particle 2) after the Universe expanded by $\mathrm{R}$ radially. This represents the maximum drag possible where Particle 1 would imitate Particle 2. The force calculation allows for Particle 1 to be pushed into that direction. The amount of drag is related to the intensity of the force between dilators.

After one de-Broglie cycle:

$$
r_{0}=\left[\begin{array}{l}
0 \\
0 \\
0 \\
R
\end{array}\right] \text { and } r-r_{0}=\Delta\left(\begin{array}{c}
\left(\frac{1}{\sqrt{3}}+\frac{v_{2}}{c} \alpha_{2}\right) \\
\left(\frac{1}{\sqrt{3}}+\frac{v_{2}}{c} \beta_{2}\right) \\
\left(\frac{1}{\sqrt{3}}+\frac{v_{2}}{c} \gamma_{2}\right) \\
0
\end{array}\right)
$$

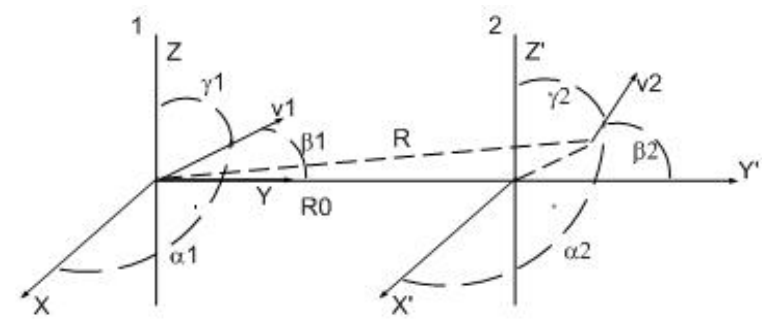

Figure 27: Derivation of Biot-Savart law using spacetime waves. 
Citation: Pereira MA (2016) The Hypergeometrical Universe: Cosmogenesis, Cosmology and Standard Model. J Generalized Lie Theory Appl 10: 248. doi:10.4172/1736-4337.1000248

Page 21 of 26

Figure 27 showcase the geometry associated with these two currents.

In the case of currents, the velocities are not relativistic and one can make the following approximations to the five-dimensional rotation matrix or metric: $\cosh \left(\alpha_{1}\right) \approx 1$ and $\sinh \left(\alpha_{1}\right) \approx v_{i} / c$ where $v_{i}$ is the velocity along the axis i. The k-vectors for the two electrons on the static reference frame are given by:

$$
k_{1}=\frac{2 \pi}{\lambda_{1}}\left[\begin{array}{llll}
\frac{1}{\sqrt{3}} & \frac{1}{\sqrt{3}} & \frac{1}{\sqrt{3}} & 1
\end{array}\right]\left[\begin{array}{cccc}
1 & 0 & 0 & -\frac{v_{1}}{c} \alpha_{1} \\
0 & 1 & 0 & -\frac{v_{1}}{c} \beta_{1} \\
0 & 0 & 1 & -\frac{v_{1}}{c} \gamma_{1} \\
\frac{v_{1}}{c} \alpha_{1} & \frac{v_{1}}{c} \beta_{1} & \frac{v_{1}}{c} \gamma_{1} & 1
\end{array}\right]
$$

Similarly:

$$
k_{2}=\frac{2 \pi}{\lambda_{2}}\left[\begin{array}{llll}
\frac{1}{\sqrt{3}} & \frac{1}{\sqrt{3}} & \frac{1}{\sqrt{3}} & 1
\end{array}\right]\left[\begin{array}{cccc}
1 & 0 & 0 & -\frac{v_{2}}{c} \alpha_{2} \\
0 & 1 & 0 & -\frac{v_{2}}{c} \beta_{2} \\
0 & 0 & 1 & -\frac{v_{2}}{c} \gamma_{2} \\
\frac{v_{2}}{c} \alpha_{2} & \frac{v_{2}}{c} \beta_{2} & \frac{v_{2}}{c} \gamma_{2} & 1
\end{array}\right]
$$

The wave intensities at $\vec{r}$ are:

$$
\begin{aligned}
& \Psi_{1}(x, y, z, r, \Phi)=\frac{\cos \left(k_{1} \cdot\left(r-r_{0}\right)\right)}{\left(1+f\left(k_{1} \cdot\left(r-r_{0}\right)\right)\right.} \\
& \Psi_{2}(x, y, z, r, \Phi)=\frac{N \cdot \cos \left(k_{2} \cdot(r-R)\right)}{\left(1+f\left(k_{2} \cdot(r-R)\right)\right)} \cong \frac{N}{\left(1+f\left(k_{2} \cdot(r-R)\right)\right)}
\end{aligned}
$$

Where $\mathrm{N}=1000$ Avogadro/, $\lambda_{1}=$ de-Broglie wavelength of a a.m.u (atomic mass unit) particle, $\lambda_{2}=$ de-Broglie wavelength of a $1 \mathrm{Kg} 4 \mathrm{D}$ particle $=\lambda_{1} / \mathrm{N}$.

To solve this optimization problem, we will find that solves this equation:

$$
\frac{d \Psi_{1}(\Delta)}{d \Delta}=\nabla \Psi_{2}(x, y, z, r, \Phi) \cdot \hat{R}
$$

this is equivalent to just summing up the rows in the gradient vector on the right-side equation. Now one can calculate:

$$
\begin{aligned}
& k_{1} \cdot\left(r-r_{0}\right)=\left(\frac{2 \pi \Delta}{\lambda_{1}}\right)\left(1+\frac{V_{1} \cdot \hat{R}}{c}+\frac{V_{2} \cdot \hat{R}}{c}+\frac{V_{1} \cdot V_{2}}{c^{2}}\right) \\
& \frac{d\left(k_{1} \cdot\left(r-r_{0}\right)\right)}{d \Delta}=\left(\frac{2 \pi}{\lambda_{1}}\right)\left(1+\frac{V_{1} \cdot \hat{R}}{c}+\frac{V_{2} \cdot \hat{R}}{c}+\frac{V_{1} \cdot V_{2}}{c^{2}}\right) \\
& \hat{R}=\frac{1}{\sqrt{3}}\left[\begin{array}{lll}
\hat{i} & \hat{j} & \hat{k}
\end{array}\right]
\end{aligned}
$$

where $\mathrm{x}[4]=0$ because $\lambda_{1}$ refers to the dilator wavelength which adjusts itself to match the de-Broglie step.

$$
\nabla f\left(k_{1} .\left(r-r_{0}\right)\right)=0 \text { due to }\left|k_{1} \cdot\left(r-r_{0}\right)\right|<<2 \pi
$$

Similarly:

$$
\begin{aligned}
& k_{2} \cdot(r-R)= \\
& =\frac{2 \pi}{\lambda_{1}}\left[\left(\frac{1}{\sqrt{3}}+\frac{v_{2}}{c} \alpha_{2}\right)\left(\frac{1}{\sqrt{3}}+\frac{v_{2}}{c} \beta_{2}\right)\left(\frac{1}{\sqrt{3}}+\frac{v_{2}}{c} \gamma_{2}\right)\left(1-\frac{v_{2}}{c} \alpha_{2}-\frac{v_{2}}{c} \beta_{2}-\frac{v_{2}}{c} \gamma_{2}\right)\right] X \\
& X=\left(\begin{array}{l}
r\left(\frac{1}{\sqrt{3}}+\frac{v_{2}}{c} \alpha_{2}\right)-\frac{R}{\sqrt{3}} \\
r\left(\frac{1}{\sqrt{3}}+\frac{v_{2}}{c} \beta_{2}\right)-\frac{R}{\sqrt{3}} \\
r\left(\frac{1}{\sqrt{3}}+\frac{v_{2}}{c} \gamma_{2}\right)-\frac{R}{\sqrt{3}} \\
0
\end{array}\right) \\
& k_{2} .(r-R) \approx \frac{2 \pi R}{\lambda_{2}}\left(1+\frac{V_{2} \cdot \hat{R}}{c}\right)
\end{aligned}
$$

where $\mathrm{x}[4]=0$ because $\lambda_{2}$ is $1 / \mathrm{N}$ of $\lambda_{1}$ and thus there is always a very close $r$ that matches a $2 \pi n$ condition. And:

$$
\begin{aligned}
& \nabla f\left(k_{2} \cdot(r-R)\right) \cong \\
& =\nabla\left(\frac{2 \pi}{\lambda_{2}}\left[\left(\frac{1}{\sqrt{3}}+\frac{v_{2}}{c} \alpha_{2}\right)\left(\frac{1}{\sqrt{3}}+\frac{v_{2}}{c} \beta_{2}\right)\left(\frac{1}{\sqrt{3}}+\frac{v_{2}}{c} \gamma_{2}\right)\left(1-\frac{v_{2}}{c} \alpha_{2}-\frac{v_{2}}{c} \beta_{2}-\frac{v_{2}}{c} \gamma_{2}\right)\right] X\right) \\
& X=\left(\begin{array}{c}
\frac{x}{\sqrt{3}}-\frac{R}{\sqrt{3}} \\
\frac{y}{\sqrt{3}}-\frac{R}{\sqrt{3}} \\
\frac{z}{\sqrt{3}}-\frac{R}{\sqrt{3}} \\
0
\end{array}\right) \\
& \nabla\left(f\left(k_{2} \cdot(r-R)\right) \cdot \hat{R}=\frac{2 \pi}{\lambda_{2}}\left(1+\frac{V_{2} \cdot \hat{R}}{c}\right)\right.
\end{aligned}
$$

Hence:

$$
\begin{aligned}
& \nabla \Psi_{1}(\Delta, \Phi) \cdot \hat{R}=-\frac{\nabla\left(k_{1} \cdot\left(r-r_{0}\right)\right)}{\left(1+f\left(k_{1} \cdot\left(r-r_{0}\right)\right)\right)} \sin \left(k_{1} \cdot\left(r-r_{0}\right)\right) \cong-k_{1} \cdot\left(r-r_{0}\right) \frac{d\left(k_{1} \cdot\left(r-r_{0}\right)\right)}{d \Delta} \\
& \nabla \Psi_{1}(\Delta, \Phi) \cdot \hat{R}=-\left(\frac{2 \pi}{\lambda_{1}}\right)^{2}\left(1+\frac{V_{1} \cdot \hat{R}}{c}+\frac{V_{2} \cdot \hat{R}}{c}+\frac{V_{1} \cdot V_{2}}{c^{2}}\right)^{2} \Delta \\
& \nabla \Psi_{2}(x, y, z, r, \Phi) \cdot \hat{R}=-\frac{N \cdot \nabla\left(k_{2} \cdot(r-R)\right)}{\left(\frac{2 \pi}{\lambda_{2}}\left(1+\frac{V_{2} \cdot \hat{R}}{c}\right) R\right)^{2}}=-\frac{N \lambda_{2}}{2 \pi} \frac{\left(1+\frac{V_{2} \cdot \hat{R}}{c}\right)}{\left(1+\frac{V_{2} \cdot \hat{R}}{c}\right)^{2}} \frac{1}{R^{2}} \\
& \nabla \Psi_{2}(x, y, z, r, \Phi) \cdot \hat{R} \cong-\frac{N \lambda_{2}}{2 \pi R^{2}\left(1+\frac{V_{2} \cdot \hat{R}}{c}\right)}
\end{aligned}
$$

Here we calculate $\Delta$ and replace it with $\frac{\Delta}{\text { NFactor }}$,$$
\frac{\Delta}{\text { NFactor }}=\frac{N \lambda_{1}{ }^{2} \lambda_{2}}{(2 \pi)^{3} R^{2}} \frac{1}{\left(1+\frac{V_{1} \cdot \hat{R}}{c}+\frac{V_{2} \cdot \hat{R}}{c}+\frac{V_{1} \cdot V_{2}}{c^{2}}\right)^{2}\left(1+\frac{V_{2} \cdot \hat{R}}{c}\right)}
$$

$$
\Delta=\frac{N \lambda_{1}^{2} \lambda_{2}}{(2 \pi)^{3} R^{2}} \frac{1}{\left(1+\frac{V_{1} \cdot \hat{R}}{c}+\frac{V_{2} \cdot \hat{R}}{c}+\frac{V_{1} \cdot V_{2}}{c^{2}}\right)\left(1+\frac{V_{2} \cdot \hat{R}}{c}\right)}
$$




$$
\Delta \cong \frac{N \lambda_{1}^{2} \lambda_{2}}{(2 \pi)^{3} R^{2}}\left\{1-\frac{V_{1} \cdot \hat{R}}{c}+\frac{\left(V_{1} \cdot \hat{R}\right)\left(V_{2} \cdot \hat{R}\right)-V_{1} \cdot V_{2}}{c^{2}}\right\}
$$

Now we will start considering attraction or repulsion components. Negative contributions are repulsion. Positive ones are attraction. In the analysis, we should consider electron-electron and electron-nuclei interactions, that is, to derive Biot-Savart Law from first principles, we should consider the dilaton waves of all components of the wire and not only the electrons. The force acting upon wire 1 is due to e1-e2 repulsion, e1-p2 attraction, p1-e2 attraction, p1-p2 repulsion, that is, there are four force components acting upon wire 1 . The same is valid for wire 2 .

$$
\begin{aligned}
& \text { Similarly: } \\
& \Delta_{e e}=-\frac{N \lambda_{1}{ }^{2} \lambda_{2}}{(2 \pi)^{3} R^{2}}\left(1-\frac{V_{1} \cdot \hat{R}}{c}+\frac{\left(V_{1} \cdot \hat{R}\right)^{2}+\left(V_{2} \cdot \hat{R}\right)^{2}+\left(V_{1} \cdot \hat{R}\right)\left(V_{2} \cdot \hat{R}\right)-V_{1} \cdot V_{2}}{c^{2}}\right) \\
& \Delta_{e p}=\frac{N \lambda_{1}^{2} \lambda_{2}}{(2 \pi)^{3} R^{2}}\left(1-\frac{V_{1} \cdot \hat{R}}{c}+\frac{\left(V_{1} \cdot \hat{R}\right)^{2}}{c^{2}}\right) \text { since } V_{2}=0 \\
& \Delta_{p e}=\frac{N \lambda_{1}^{2} \lambda_{2}}{(2 \pi)^{3} R^{2}}\left(1+\frac{\left(V_{2} \cdot \hat{R}\right)^{2}}{c^{2}}\right) \text { since } V_{1}=0 \\
& \Delta_{p p}=-\frac{N \lambda_{1}^{2} \lambda_{2}}{(2 \pi)^{3} R^{2}}(1) \text { since } V_{1}=V_{2}=0 \\
& \Delta_{\text {total }}=\Delta_{e e}+\Delta_{e p}+\Delta_{p e}+\Delta_{p p}=\frac{N \lambda_{1}^{2} \lambda_{2}}{(2 \pi)^{3} R^{2}} \frac{\left(V_{1} \cdot \hat{R}\right)\left(V_{2} \cdot \hat{R}\right)-V_{1} \cdot V_{2}}{c^{2}}
\end{aligned}
$$

Where $\mathrm{p}$ stands for proton and e for electron.

$$
r=\frac{N \lambda_{1}{ }^{2} \lambda_{2}}{(2 \pi)^{3}}\left(\frac{V_{2} \cdot \hat{R}}{c} \frac{V_{1} \cdot \hat{R}}{c}-\frac{V_{1} \cdot V_{2}}{c^{2}}\right) \frac{\hat{R}}{R^{2}}
$$

Using $\mathrm{a} \times(\mathrm{b} \times \mathrm{c})=\mathrm{b}(\mathrm{a} . \mathrm{c})-\mathrm{c}(\mathrm{a} \cdot \mathrm{b})$ :

$$
r=\frac{N \lambda_{1}^{2} \lambda_{2}}{(2 \pi)^{3} c^{2}}\left(V_{1} \otimes\left(V_{2} \otimes \hat{R}\right)\right) \frac{\hat{R}}{R^{2}}
$$

The number of dilators can be $1000^{\star}$ Avogadro/ $\chi$ for $\mathrm{Kg} 4 \mathrm{D}$ or $\mathrm{Kg} 3 \mathrm{D}$, or 1/e for a Coulomb:

The force between two $1 \mathrm{Kg} 3 \mathrm{D}$ dilators separated by 1 meter is given by:

$$
\begin{aligned}
& F=m_{03 D} c^{2} \frac{d \tan \left(\alpha_{\tau}\right)}{d \tau}=(K g 3 D)\left(\frac{N}{\chi}\right) c^{2} \frac{r}{\lambda_{1}^{2}} \\
& F=\left(\frac{N}{\chi}\right)^{2} \frac{\lambda_{2}}{(2 \pi)^{3}(K g 3 D)}\left(V_{1} \otimes\left(V_{2} \otimes \hat{R}\right)\right) \frac{(\text { Kg } 3 D)^{2}}{(\text { meter })^{2}}
\end{aligned}
$$

To scale this force into the force between two Coulomb charges traveling with velocities $v_{1}$ and $v_{2}$ one just have to multiply the equation by $(1 \mathrm{C} / \mathrm{Ne})^{2}$ :

$$
\begin{gathered}
F=\left(\frac{1 \text { Coulomb }}{e}\right) c^{2} \frac{r}{\lambda_{1}^{2}}=\left(\frac{1 \text { Coulomb }}{e}\right)^{2} \frac{\lambda_{2} v_{1} \cdot v_{2}}{(2 \pi)^{3}}\left(\left(d l_{1} \otimes\left(d l_{2} \otimes \hat{R}\right)\right)\right) \frac{\hat{R}}{R^{2}}= \\
=\left(\frac{1 \text { Coulomb }}{e}\right)^{2} \frac{\lambda_{2} j_{1} \cdot j_{2}(K g 3 D)}{(2 \pi)^{3} e^{2}}\left(\left(d l_{1} \otimes\left(d l_{2} \otimes \hat{R}\right)\right)\right) \frac{\vec{R}}{R^{3}}
\end{gathered}
$$

The Biot-Savart law can be written as:

$$
d F=\frac{\mu_{0} I_{1} I_{2}}{4 \pi} \frac{\left(d l_{1} \cdot d l_{2}\right)}{\left|\vec{x}_{12}\right|^{3}} \vec{x}_{12}
$$

Comparing the two equations one obtains:

$$
\frac{\mu_{0}}{4 \pi}=\frac{\lambda_{2}(1 K g 3 D)}{(2 \pi)^{3} e^{2}}
$$

Thus

$\mu_{0}=\frac{\lambda_{2}(K g 3 D)}{2 \pi^{2} e^{2}}$

Without that extra unit $(\mathrm{Kg} 3 \mathrm{D}), \mu_{0}$ will not have the appropriate units. The same is valid for $\varepsilon_{0}$.

From equation (52)

$\varepsilon_{0}=\frac{2 \pi^{2} e^{2}}{\lambda_{2} c^{2}(K g 3 D)}$

Thus

$$
\mu_{0} \cdot \varepsilon_{0}=\frac{\lambda_{2}(K g 3 D)}{2 \pi^{2} e^{2}} \frac{2 \pi^{2} e^{2}}{\lambda_{2} c^{2}(K g 3 D)}=\frac{1}{c^{2}}
$$

Thus one recovers the relationship between $\varepsilon_{0}$ and $\mu_{0}$. We recovered the Biot-Savart law for infinitesimal elements of current. This was achieved by considering the many contributions of positive and negative center charges and using the low velocity approximation. All dilators contribute to electromagnetism and not just electrons.

Within a Tokamak Nuclear Fusion device, currents are both positive and negative (hot plasma) and velocities are relativistic. Under these conditions one should use the non-approximated first half identity from equation

$$
\begin{aligned}
& \Delta=\frac{N \lambda_{1}^{2} \lambda_{2}}{(2 \pi)^{3} R^{2}} \frac{1}{\left(1+\frac{V_{1} \cdot \hat{R}}{c}+\frac{V_{2} \cdot \hat{R}}{c}+\frac{V_{1} \cdot V_{2}}{c_{1}}\right)\left(1+\frac{V_{2} \cdot \hat{R}}{c}\right)} \\
& \frac{\Delta F}{N F} \frac{N \lambda_{1}{ }^{2} \lambda}{(2 \pi)^{3} R^{2}} \frac{1}{\left(1+\frac{V_{1} \cdot \hat{R}}{c}+\frac{V_{2} \cdot \hat{R}}{c}+\frac{V_{1} \cdot V_{2}}{c^{2}}\right)^{2}\left(1+\frac{V_{2} \cdot \hat{R}}{c}\right)} \\
& \Delta=\frac{N \lambda_{1}^{2} \lambda_{2}}{(2 \pi)^{3} R^{2}} \frac{1}{\left(1+\frac{V_{1} \cdot \hat{R}}{c}+\frac{V_{2} \cdot \hat{R}}{c}+\frac{V_{1} \cdot V_{2}}{c^{2}}\right)\left(1+\frac{V_{2} \cdot \hat{R}}{c}\right)} \\
& \Delta \cong \frac{N \lambda_{1}{ }^{2} \lambda_{2}}{(2 \pi)^{3} R^{2}}\left\{1-\frac{V_{1} \cdot \hat{R}}{c}+\frac{\left(V_{1} \cdot \hat{R}\right)\left(V_{2} \cdot \hat{R}\right)-V_{1} \cdot V_{2}}{c^{2}}\right\}
\end{aligned}
$$

The force between two $1 \mathrm{Kg} 4 \mathrm{D}$ dilators is given by:

$$
\begin{aligned}
& F=m_{03 D} c^{2} \frac{d \tan \left(\alpha_{\tau}\right)}{d_{\tau}}=(K g 3 D)\left(\frac{N}{\chi}\right) c^{2} \frac{r}{\lambda_{1}^{2}} \quad(151) \\
& F=(K g 3 D)\left(\frac{N}{\chi}\right)^{2} \frac{\lambda_{2}}{(2 \pi)^{3} R^{2}} \frac{1}{\left(1+\frac{V_{1} \cdot \hat{R}}{c}+\frac{V_{2} \cdot \hat{R}}{c}+\frac{V_{1} \cdot V_{2}}{c^{2}}\right)\left(1+\frac{V_{2} \cdot \hat{R}}{c}\right)} \hat{R}
\end{aligned}
$$

To scale this force into the force between two Coulomb charges traveling with velocities $v_{1}$ and $v_{2}$ one just have to multiply the equation by $\left(\frac{1 C}{N e}\right)^{2}$ :

$$
\begin{aligned}
& F=\frac{\mu_{0} c^{2} C_{1} C_{2}}{4 \pi} \frac{1}{\left(1+\frac{V_{1} \cdot \hat{R}}{c}+\frac{V_{2} \cdot \hat{R}}{c}+\frac{V_{1} \cdot V_{2}}{c^{2}}\right)\left(1+\frac{V_{2} \cdot \hat{R}}{c}\right)} \frac{\hat{R}}{R^{2}} \\
& F=\frac{C_{1} C_{2}}{4 \pi \varepsilon_{0}} \frac{1}{\left(1+\frac{V_{1} \cdot \hat{R}}{c}+\frac{V_{2} \cdot \hat{R}}{c}+\frac{V_{1} \cdot V_{2}}{c^{2}}\right)\left(1+\frac{V_{2} \cdot \hat{R}}{c}\right)} \frac{\hat{R}}{R^{2}}
\end{aligned}
$$


Where $\mathrm{C} 1$ and $\mathrm{C} 2$ are charges traveling at $v_{1}$ and $v_{2}$ and $\mathrm{c}$ is the speed of light. The asymmetry with respect to $v_{2}$ i s the not lost in our considerations. Now, we will not dwell on it since the small velocity approximation matched Biot-Savart Law and we also recovered the Gerber Potential (thus recovering the Mercury Perihelion Precession Measurements). In addition, there are experimental results by NASA (EM Drive) that indicate the possibility of propulsion based on electromagnetic fields (moving charges) and that might be related to these results.

Gyrogravitation-electromagnetism unification: Similarly, one can derive the Gravitational Biot-Savart equation by simple analogy to our derivation of the Gravitation Law.

The limit with zero velocity independent term corresponds to the steady state gravitational field (Newton's Law).

$$
\begin{aligned}
& F_{\text {Gravitational }}=\left[\left(\frac{N}{\chi}\right) \frac{\lambda_{1} c^{2}}{(2 \pi)^{3}} \frac{\lambda_{1}}{R_{0}} \xi\right] M_{1} M_{2} \frac{1}{\left(1+\frac{V_{1} \cdot \hat{R}}{c}+\frac{V_{2} \cdot \hat{R}}{c}+\frac{V_{1} \cdot V_{2}}{c^{2}}\right)\left(1+\frac{V_{2} \cdot \hat{R}}{c}\right)} \frac{\hat{R}}{R^{2}} \\
& G=\left[\left(\frac{N}{\chi}\right) \frac{\lambda_{1} c^{2}}{(2 \pi)^{3}} \frac{\lambda_{1}}{R_{0}} \xi\right] \\
& F_{\text {Gravitational }}=\left[\left(\frac{N}{\chi}\right) \frac{\lambda_{1} c^{2}}{(2 \pi)^{3}} \frac{\lambda_{1}}{R_{0}} \xi\right] M_{1} M_{2} \frac{\hat{R}}{R^{2}}
\end{aligned}
$$

Notice that the value of the Gravitational Constant $\mathrm{G}$ is inversely proportional to the $4 \mathrm{D}$ Radius of the Universe $R_{0}$. This means that at earlier epochs, Gravitation was stronger and at a precise time in the life of the Universe all forces had the same strength. It also means that Stellar Candles would contain smaller masses in the past than they do at later epochs. This means that current measurements of distances across the Universe based upon Stellar Candles might not work properly and indicate unreasonable large distance incompatible with the age of the Universe.

For non-zero relative speed, we obtain the Hypergeometrical Universe Law of Gravitation (see Appendix):

$$
\begin{aligned}
& F=G M_{1} M_{2} \frac{1}{\left(1+\frac{V_{1} \cdot \hat{R}}{c}+\frac{V_{2} \cdot \hat{R}}{c}+\frac{V_{1} \cdot V_{2}}{c^{2}}\right)\left(1+\frac{V_{2} \cdot \hat{R}}{c}\right)} \frac{\hat{R}}{R^{2}} \\
& F \cong G M_{1} M_{2} \frac{\hat{R}}{R^{2}}\left(1-\frac{V_{1} \cdot \hat{R}}{c}+\frac{\left(V_{1} \cdot \hat{R}\right)^{2}+\left(V_{2} \cdot \hat{R}\right)^{2}+\left(V_{1} \cdot \hat{R}\right)\left(V_{2} \cdot \hat{R}\right)-V_{1} \cdot V_{2}}{c^{2}}\right)
\end{aligned}
$$

Equations (96) and (97) express the force for two elements of charge in motion. They recover Gauss Law under conditions of rest and have identical form as equation (98). This means that a single equation describes everything we know about electrostatics, electromagnetism and gravitation.

The Force derivation uses a boundary condition where the dilator is at rest with respect to the FS. This is equivalent to say that all forces are partial derivatives with respect to $\mathrm{R}$ while keeping velocity constant. This is important since the force is velocity dependent. To obtain a potential from which one can calculate dynamics, one need to integrate the equation (101) with respect to $R$.

$$
V_{2}\left(R, V_{1}, V_{2}\right)=\frac{G M_{1}}{\left(1+\frac{V_{1} \cdot \hat{R}}{c}+\frac{V_{2} \cdot \hat{R}}{c}+\frac{V_{1} \cdot V_{2}}{c^{2}}\right)\left(1+\frac{V_{2} \cdot \hat{R}}{c}\right)} \frac{1}{R}
$$

This equation was derived under the regimen of weak (normal) gravitational pull. It would be easy to derive the same equation for conditions in the surroundings of a Black Hole. One would just not use the derivative approximations.

This means that there is Antigravity (weakening of Gravitation) right within the Law of Gravitation. If for a moment one sets the referential frame on body 1 , thus having $\mathrm{V} 1=0$, the Gravitational Force on $\mathrm{F} 2$ becomes:

$$
F_{2}=G M_{1} M_{2} \frac{1}{\left(1+\frac{V_{2} \cdot \hat{R}}{c}\right)^{2}} \frac{\hat{R}}{R^{2}} \approx G M_{1} M_{2} \frac{\hat{R}}{R^{2}}\left(1-2\left(\frac{V_{2} \cdot \hat{R}}{c}\right)+3\left(\frac{V_{2} \cdot \hat{R}}{c}\right)^{2}-4\left(\frac{V_{2} \cdot \hat{R}}{c}\right)^{3} \ldots\right)
$$

This is a much more complex view of Gravitation and it is a view derived from a more fundamental model. It reduces to Newton's Law at zero relative velocity. This might be involved in the production of double jets in Black Holes (Figures 28-34).

\section{Conclusions}

The Hypergeometrical Universe theory, a model that considers the interference of four-dimensional wave on the hypersurface of a hyperspherical expanding universe was introduced. The complexity of the present description of the universe in our sciences is assigned to the fact that one is dealing with four-dimensional projections of a fivedimensional process. Our inability to realize that made the description unnecessarily complex.

These are the ingredients for a new and simple formulation of Physics:

1. A new quantum Lagrangian principle (QLP) was proposed.

2. Quantum gravity, electrostatics and electromagnetism were derived using the same equations (QLP), same framework. The theory is inherently quantum mechanical and relativistic.

3. A new Force of Nature (de-Broglie Force) was recognized. Strong and Weak Forces were deemed unnecessary.

4. The quantum version of this theory is readily achieved just by eliminating the high mass or short wavelength approximation on equation (27). It is outside the scope of this paper to implement Hypergeometrical Universe Quantum Algorithms. In a fully geometric theory, there are no energy or mass quanta. Motion is quantized by the QLP. All the other quantizations can be recovered from that.

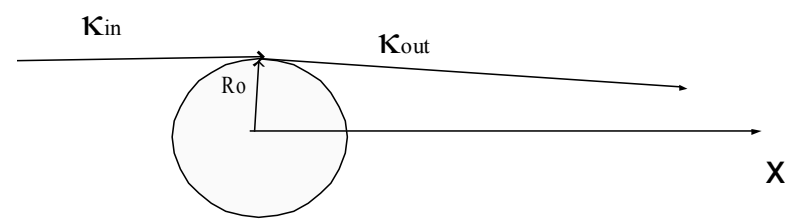

Figure 28: Gravitational induced scattering due to Gravitational Force acting upon a photon.

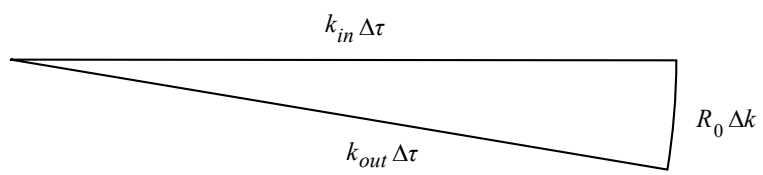

Figure 29: Phase-matching condition on Gravitational Lensing event 


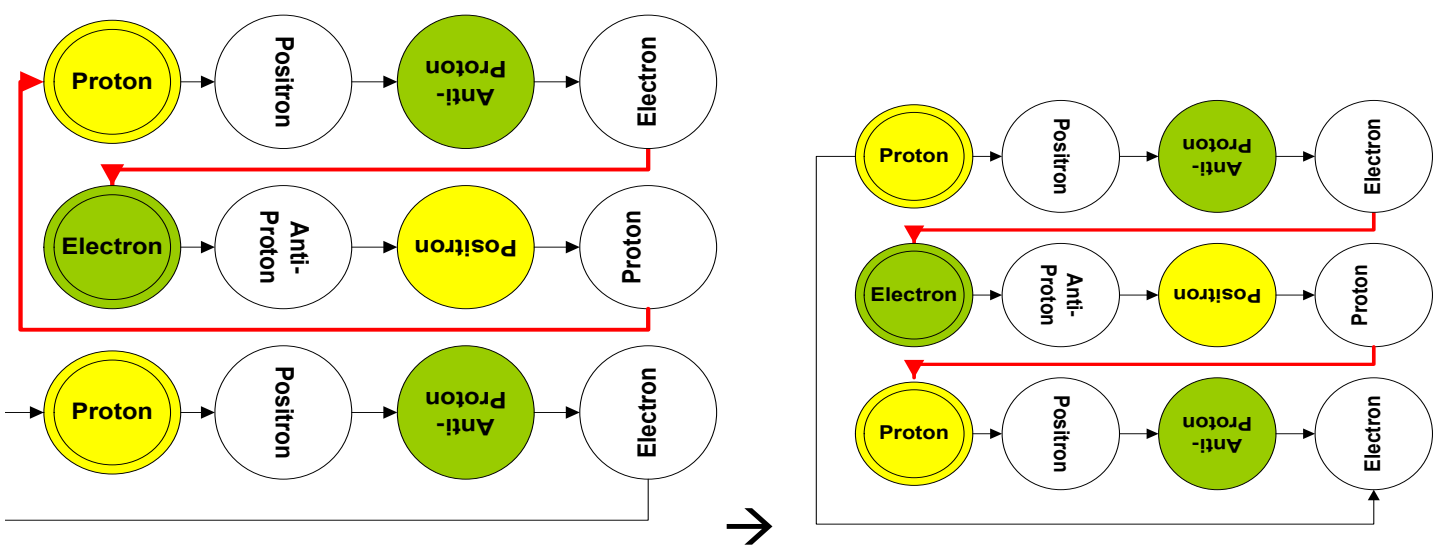

Figure 30: Deuterium diagram.

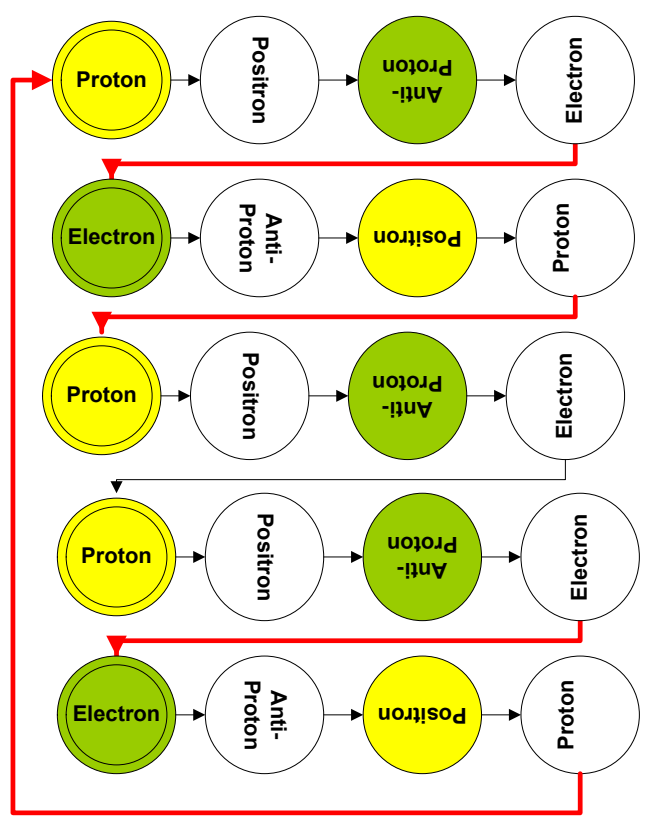

Figure 31: Tritium diagram.

The Hypergeometrical Universe Model provides alternative views on matter and forces by changing the paradigm under which to describe events. The model provides an alternative Standard Model, Cosmology, Cosmogenesis while maintaining compatibility with Relativity and Quantum Mechanics. The Fundamental Dilator together with the Lightspeed Expanding Universe and the Quantum Lagrangian Principle provides the basis for Quantum Mechanics.

Two fundamental parameters of the universe were calculated from the first principles (permittivity and magnetic susceptibility of vacuum). Universal Gravitational Constant $\mathrm{G}$ was also calculated from first principles. $\mathrm{G}$ was proposed to be inversely proportional to the $4 \mathrm{D}$ radius of the Universe and used to explain implausible Stellar Candle readings.

HU Supernova Analysis brought to light that ancient photons should slow down as they approach us and that the luminosity decay is not dependent directly solely on distance. It is dependent on the

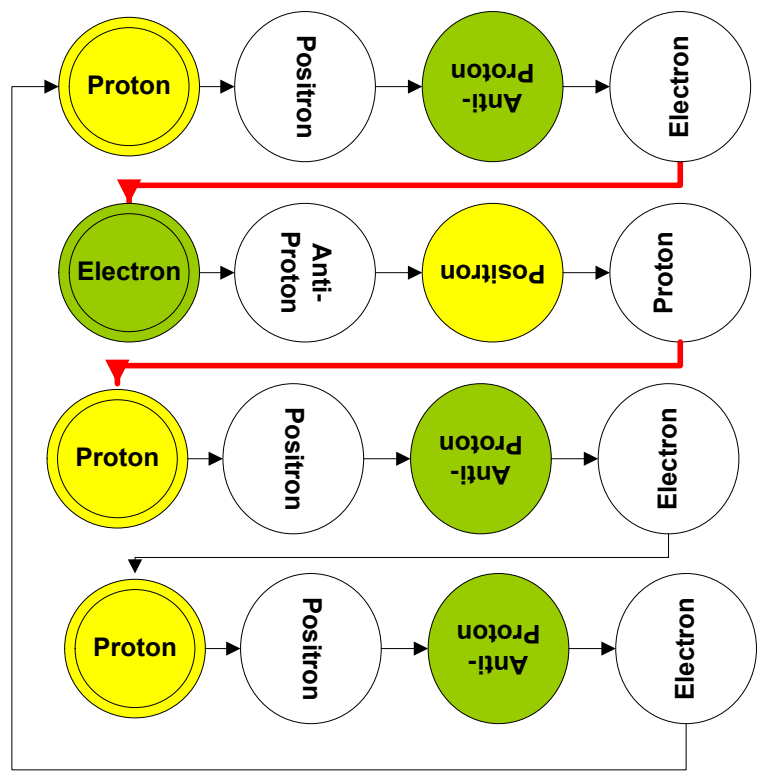

Figure 32: Helium 3 diagram.

number of cycles the dilaton field went through. That number of cycles is the same, no matter where you are within a hypersphere or epoch.

Using the Quantum Lagrangian Principle to model dynamics naturally bring about the observed speed of light as being the maximum speed in this Universe. It also explains the reason for increased inertial mass and the slowing down of time with speed (increase twisting of local FS). The larger the speed (local FS twist), the smaller the effect of subsequent interactions (accelerations) will be. This allows for a new understanding of the passage of time.

Nuclear lifetimes are also affected by the local twisting. A more detailed analysis is outside the scope of this paper and will be presented elsewhere [10].

The concept of the Fundamental Dilator brings about a view of a Stroboscopic Universe where interaction is intermittent and where particle substructure is easily explained by the polymeric nature of dilator coherences. It also brings about the possibility of thinking of matter in terms of metric deformations, thus capable of beating and 

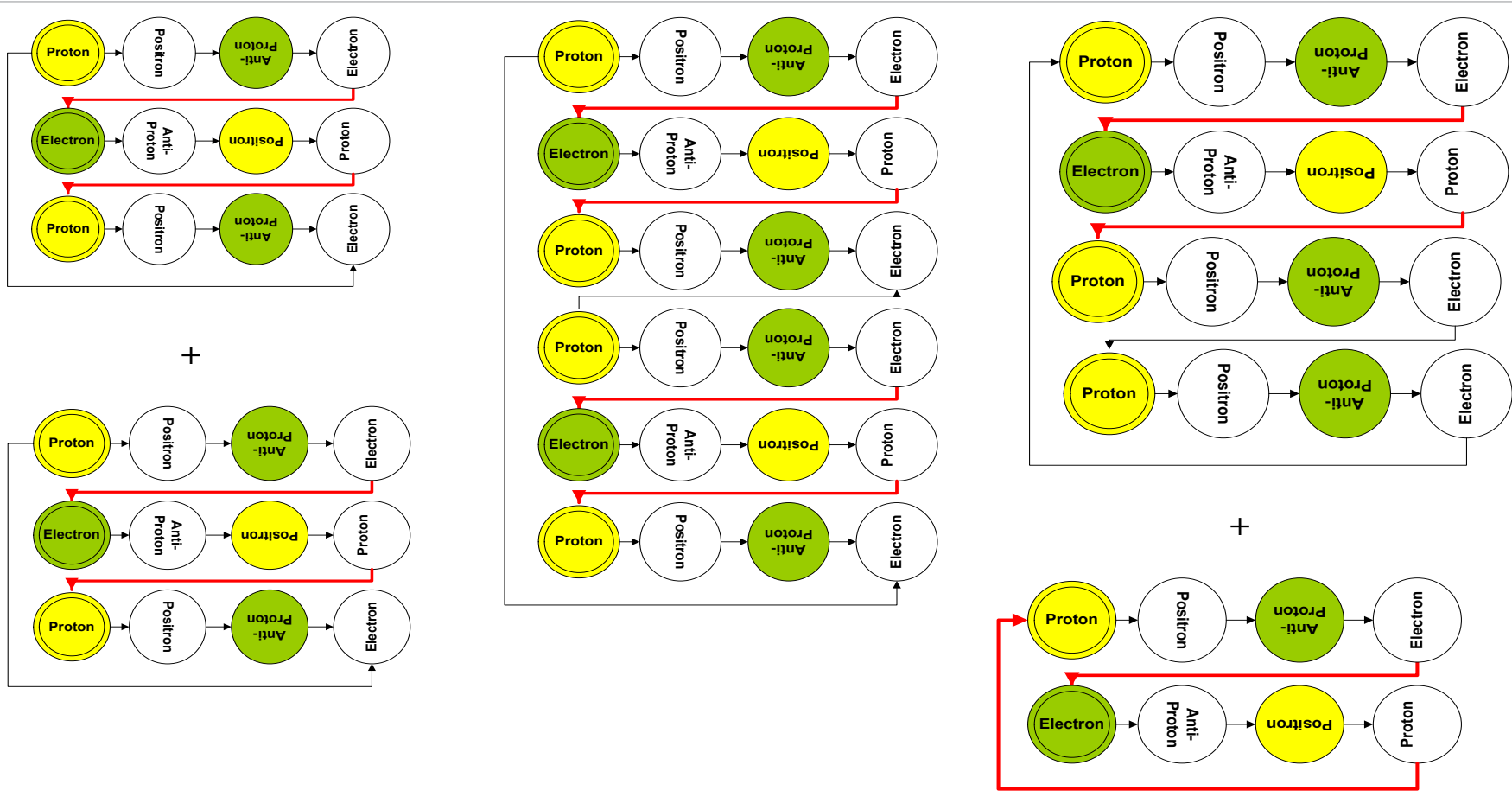

Figure 33: Nuclear Fusion diagram.
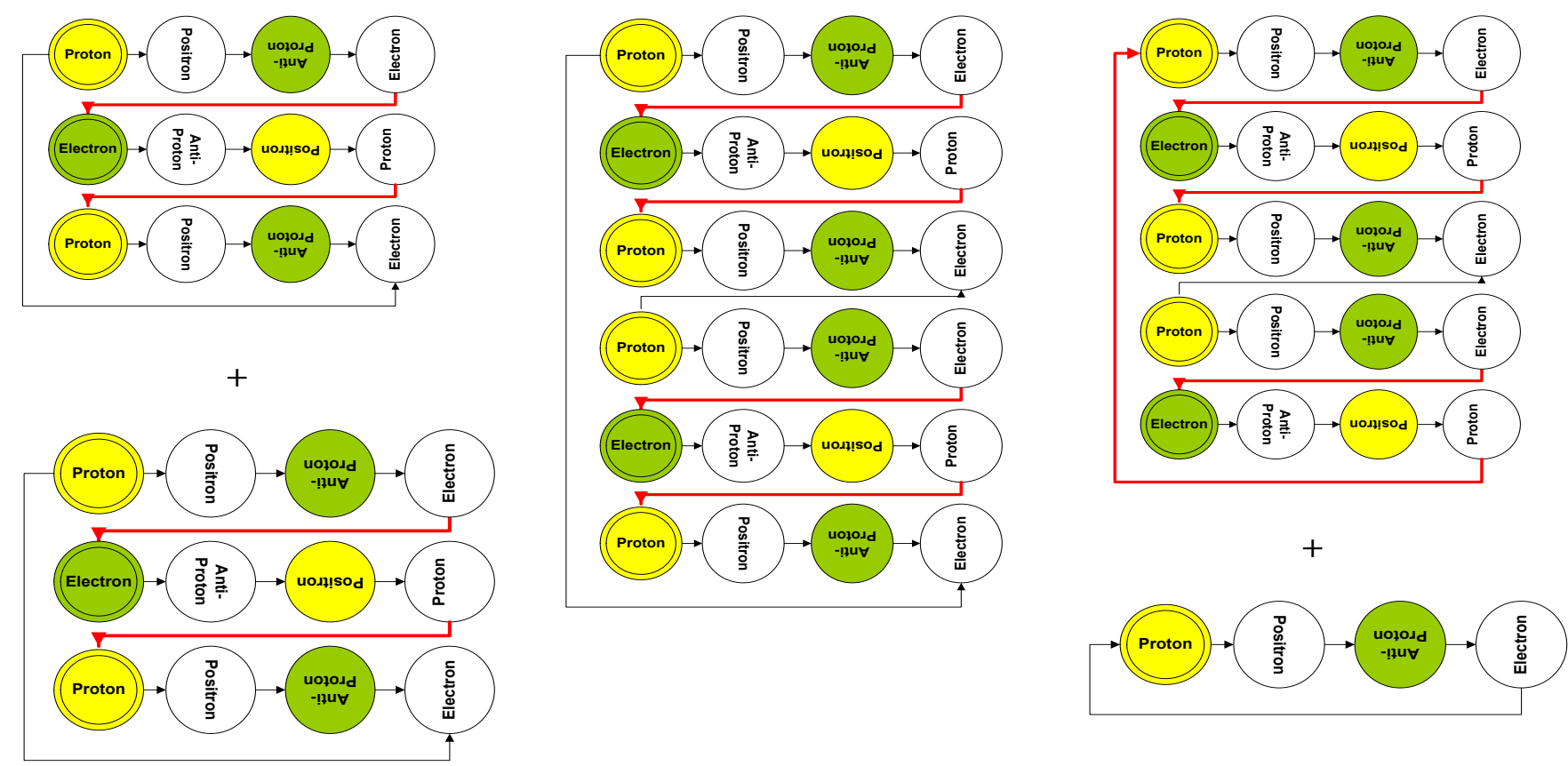

Figure 34: Nuclear Fusion products diagram

nonlinear hadronic processes. We proposed new experiments that might bring about Coherent Nuclear Fusion along the lines of nonlinear optical interactions. Phase matching angle for coherent hadronic processes is tuned by changing the relative interaction velocity, which is an angle or direction in the $4 \mathrm{D}$ spatial manifold.

Gyrogravitation and Electromagnetism Natural Laws were derived from first principles.
The theory was applied to standard tests (Precession of Mercury Perihelion, Gravitational Lensing), was used to explain the Stellar Candle paradox without the use of inflation, Hubble expansion without Dark Energy, Neutron Decay without Electroweak Interaction, Particle Substructure without quark composition and Black Hole's Double Jets with the use of Gyrogravitation. It also provides a potential solution to the Spiral Galaxy rotation problem without the need for Dark Matter. 
Citation: Pereira MA (2016) The Hypergeometrical Universe: Cosmogenesis, Cosmology and Standard Model. J Generalized Lie Theory Appl 10: 248. doi:10.4172/1736-4337.1000248

Page 26 of 26

The Fabric of Space Stress-Strain paradigm applied to the two cross-sections of the Universe (RXYZ and $\Phi X Y Z$ allowed for the derivation from first principles of natural laws (Gauss, Biot-Savart, Newton's Gravitation) and the derivation of a more general equation that applies to all forces.

Four Newton's Laws were recast in terms of Stress-Strain relationships. The theory also explains why things move inertially and why the speed of light is a limit.

A new paradigm for Dark Matter, antimatter from a Lagging Hypersphere was proposed.

\section{De Broglie force, the two-slit experiment and quantum mechanics}

The theory proposes a new Force de-Broglie force by providing a dilaton representation of matter waves. This new force can be used to control particles in the same way as electromagnetism or gravitation, with implications on accelerator design and coherent nuclear fusion reactor architecture. It also explains the self-interference process occurring in a two-slit experiment. The particle rides (and creates) a de-Broglie dilaton field. The particle enters one slit while the dilaton field enter both. The exiting dilaton field from both slits, interfere and guide the particle into the detector in an interferometric pattern. Thus, the electron passing through the two slits is not a good example of a Quantum Process and there is no need to invoke particle-wave duality. Instead, it is a wave-generator, wave interaction process.

This view parallels de-Broglie-Bohm theory with the distinction that wavefunctions are assigned a physical meaning (a propagating deformation of space or the Fabric of Space) and that consideration gives rise to the discovery of a new Force in Nature, so despite of similarities, the perspective unveiled in this theory is distinct and more profound. On the other hand, de-Broglie Bohm theory dynamics equations fails to provide a comparative framework associating deBroglie Force Field and other dilatons fields (electromagnetism). They are free particle equations.

The Hypergeometrical Universe also provides an alternative interpretation for the Quantum Mechanics wavefunction as the loci associated with each footprint of the dilators as the Universe expands.

Quantum Mechanics should be recovered from the Hypergeometrical Universe Fundamental Equation without the largenumber-of-dilators approximation. It points to a new interpretation of Quantum Mechanics Wavefunctions based on Lissajous interferences in a $5 \mathrm{D}$ Spacetime.

\section{Summary}

This is a simple theory in terms of formalism, which provides new insights, proven and testable predictions. A simple mapping from HU Fundamental Dilator based hyperons and isotopes to the Standard Model might be enough to reconcile $\mathrm{HU}$ to Quantum Chromodynamics. This new view of reality together with the proposition of the new deBroglie Force might provide simple solutions to currently intractable problems.

\section{References}

1. Gerber $P$ (1898) Die räumliche und zeitliche Ausbreitung der Gravitation (Space and temporary propagation of gravitation). Z Math Phys 43: 93-104.

2. Friedman $A$ (1922) Über die Krümmung des Raumes. Z Phys (in German) 10 377-386.

3. Pereira M, Hypergeometrical Universe Theory Supernovae High Z Predictions submitted to Science Direct, Helyon.

4. Schwarzschild B (2003) WMAP Spacecraft Maps the Entire Cosmic Microwave Sky With Unprecedented Precision. Physics Today 56: 21

5. Gerber P (1917) Die Fortpflanzungsgeschwindigkeit der Gravitation. (The propagation-velocity of gravitation). Ann Phys 52: 415-444.

6. Pauli W (1958) Theory of Relativity. London: Pergamon Press.

7. Landau LD, Lifshitz EM (1975) The Classical Theory of Fields. Oxford Pergamon Press.

8. Jackson JD (1975) Classical Electrodynamics. John Wiley \& Sons.

9. Edwin H (1929) A relation between distance and radial velocity among extragalactic nebulae. PNAS. 15: 168-173.

10. Lach J (1985) Hyperon Polarization and Magnetic Moments, FermilabConf-93/381.
Citation: Pereira MA (2016) The Hypergeometrical Universe: Cosmogenesis, Cosmology and Standard Model. J Generalized Lie Theory Appl 10: 248. doi:10.4172/1736-4337.1000248
OMICS International: Open Access Publication Benefits \&

\section{Features}

Unique features:

- Increased global visibility of articles through worldwide distribution and indexing

- Showcasing recent research output in a timely and updated manner

Special issues on the current trends of scientific research

Special features:

- 700+ Open Access Journal

$50,000+$ editorial team

Rapid review proces

Quality and quick editorial, review and publication processing

Indexing at major indexing services

Sharing Option: Social Networking Enabled

Authors, Reviewers and Editors rewarded with online Scientific Credits

Better discount for your subsequent articles

Submit your manuscript at: http://www.omicsonline.org/submission 